\title{
Topological Structure of Manufacturing Industry Supply Chain Networks
}

\author{
Supun S. Perera $\mathbb{D}^{1},{ }^{1}$ Michael G. H. Bell, ${ }^{1}$ Mahendrarajah Piraveenan, ${ }^{2}$ \\ Dharshana Kasthurirathna, ${ }^{3}$ and Mamata Parhi ${ }^{4}$ \\ ${ }^{1}$ Business School Institute of Transport and Logistics Studies (ITLS), University of Sydney, Australia \\ ${ }^{2}$ School of Civil Engineering Complex Systems Research Group (CSRG), University of Sydney, Australia \\ ${ }^{3}$ Faculty of Computing, Sri Lanka Institute of Information Technology (SLIIT), Sri Lanka \\ ${ }^{4}$ Roehampton Business School, University of Roehampton, UK
}

Correspondence should be addressed to Supun S. Perera; supun.perera@sydney.edu.au

Received 19 February 2018; Revised 29 April 2018; Accepted 8 August 2018; Published 3 October 2018

Academic Editor: Nikos Papakostas

Copyright $\odot 2018$ Supun S. Perera et al. This is an open access article distributed under the Creative Commons Attribution License, which permits unrestricted use, distribution, and reproduction in any medium, provided the original work is properly cited.

Empirical analyses of supply chain networks (SCNs) in extant literature have been rare due to scarcity of data. As a result, theoretical research have relied on arbitrary growth models to generate network topologies supposedly representative of realworld SCNs. Our study is aimed at filling the above gap by systematically analysing a set of manufacturing sector SCNs to establish their topological characteristics. In particular, we compare the differences in topologies of undirected contractual relationships (UCR) and directed material flow (DMF) SCNs. The DMF SCNs are different from the typical UCR SCNs since they are characterised by a strictly tiered and an acyclic structure which does not permit clustering. Additionally, we investigate the SCNs for any self-organized topological features. We find that most SCNs indicate disassortative mixing and power law distribution in terms of interfirm connections. Furthermore, compared to randomised ensembles, self-organized topological features were evident in some SCNs in the form of either overrepresented regimes of moderate betweenness firms or underrepresented regimes of low betweenness firms. Finally, we introduce a simple and intuitive method for estimating the robustness of DMF SCNs, considering the loss of demand due to firm disruptions. Our work could be used as a benchmark for any future analyses of SCNs.

\section{Introduction}

Due to the increasingly complex and interconnected nature of the global supply chain networks (SCNs), recent research has focussed on modelling supply chains as complex adaptive systems using network science concepts [1,2]. Following on from the work published by [3], who used network science techniques to generate a network topology and investigate its topological robustness, a large number of theoretical research papers have appeared in this area [4-12]. These studies have theoretically formulated generalizable growth mechanisms underlying the firm-partnering process in SCN formation. Subsequently, the network topologies generated based on various growth models have been investigated for their topological characteristics, such as robustness and efficiency.
Despite the large number of theoretical papers published within the past few years on network modelling of SCNs, the effort on empirical validation of the theoretical findings has been limited. This is mainly due to difficulty in obtaining large-scale datasets on supplier-customer relationships, which are often proprietary and confidential. Papers which systematically analyse the topologies of real-world SCNs, the conclusions of which can then be used to inform modelling efforts, have been relatively scarce.

In light of the above, this study presents a comprehensive analysis of two sets of SCN datasets, namely,

(1) The dataset of Indian automobile manufacturers [13]. This SCN includes contractual relationships between various firms (therefore it is modelled as an undirected network). 
(2) Twenty-six SCNs across various manufacturing industry sectors, based on the dataset presented in Willems [14]. These SCNs include material flows between firms (as such, it is modelled as a directed network). All the SCNs in this dataset include the full depth in terms of tiers (from suppliers to retailers).

In particular, this study is aimed at addressing the following key research questions pertaining to SCN topology and robustness:

(1) What common topological characteristics (if any) can be expected from the directed material flow SCNs in the manufacturing sector?

(2) What are the key differences (if any) in the topology of directed material flow and undirected contractual relationship SCNs?

(3) Are there any self-organized features present in any of the SCNs?

(4) Are there any correlations between node attributes and the topological features of nodes in the directed material flow SCNs?

(5) How can we determine the robustness of SCNs, considering the inherent differences between the contractual relationship and material flow networks?

The remainder of this manuscript is structured as follows. Section 2 provides the background to this study and introduces key theoretical concepts in terms of network topological analysis. Section 3 describes the structure and limitations of each dataset considered, while Section 4 presents the data analysis methodology and results. Section 5 provides a discussion of the results obtained, and Section 6 concludes the paper.

\section{Background}

2.1. Topology of Undirected and Directed SCNs. The interfirm relationships in SCNs are commonly modelled using undirected links. However, the links between nodes in a SCN can include a direction, depending on the specific type of relationship being modelled. The interfirm relationships in a SCN can be broadly categorised into three classes, namely, (1) material flows, (2) financial flows, and (3) information exchanges. Material flows are usually unidirectional from suppliers to retailers, while financial flows are unidirectional in the opposite direction. Both material and financial flows mostly occur vertically, across the functional tiers of a SCN (however, in some cases, two firms within the same tier, such as two suppliers, could also exchange material and finances) [15]. In contrast, information exchanges are bidirectional (i.e., undirected) and include both vertical and horizontal connections (i.e., between firms across tiers and between firms within the same tier). Therefore, the same SCN can include different topologies based on the specific type of relationship denoted by the links in the model. For instance, unlike material and financial flows, SCN topology for information exchanges can exhibit shorter path lengths and high clustering due to a relatively larger number of horizontal connections [16].

Compared to undirected network representation, in directed networks, the adjacency matrix is no longer symmetric. As a result, the degree of a node in a directed network is characterised by both in-degree and out-degree. On this basis, the degree distribution of directed networks is analysed separately for in- and out-degrees. Also, unlike undirected networks, in directed networks the distance between node $i$ and node $j$ is not necessarily the same as the distance between node $j$ and node $i$. In fact, in directed networks, the presence of a path from node $i$ to node $j$ does not necessarily imply the presence of a path from node $j$ to node $i$ [17]. This has implications on node centrality metrics, such as closeness and betweenness. In addition, many dynamics such as synchronizability and percolation are different in directed networks compared to undirected networks [18, 19]. Therefore, when modelling SCNs, it is important to first identify the specific type of relationship denoted by the links, so that the network can be correctly represented as undirected or directed.

In this paper, we consider two types of SCNs, namely, (1) the SCN of Indian automobile manufacturers, which is modelled as an undirected network (referred to as the undirected contractual relationship SCN or UCR-SCN throughout this paper) and (2) twenty-six material flow SCNs across various manufacturing industry sectors, based on the dataset presented in Willems [14], which are modelled as directed networks (referred to as the directed material flow SCN or DMF-SCN throughout this paper).

2.2. Characterising SCNs Using Network Science Metrics. Mathematical analysis of a network requires it to be represented through an adjacency matrix $(A)$. An element $A_{i j}$ of the adjacency matrix $A$, for an undirected network, is given as

$$
A_{i j}= \begin{cases}1 & \text { if there is a link between nodes } i \text { and } j \\ 0 & \text { otherwise. }\end{cases}
$$

In contrast, an element of the adjacency matrix, for a directed network, is given as

$$
A_{i j}= \begin{cases}1 & \text { if there is a link from node } j \text { to node } i \\ 0 & \text { otherwise. }\end{cases}
$$

The key difference between the adjacency matrix of an undirected and a directed network is that the adjacency matrix of an undirected network has two entries for each link (i.e., the adjacency matrix of undirected networks is symmetric). Therefore, the total number of links $(L)$ for an undirected network is calculated as $L=1 / 2 \sum_{i j} A_{i j}$, while for a directed network it is calculated as $L=\sum_{i j} A_{i j}$.

In this paper, we have modelled all SCNs as unweighted networks where nodes represent individual firms. For the UCR-SCN, the links represent undirected 
contractual relationships between firms, while in the DMF-SCNs, the links represent the directed material flows between firms.

The metrics used to characterise the topology of complex networks can be classified into node- and networklevel metrics (see Costa et al. [20] and Rubinov and Sporns [21] for a comprehensive range of measurements used for characterization of complex networks). Table 5 in Appendix A presents the list of network-level metrics used for analysis in this study, and their implications within a SCN context.

Node-level metrics characterise, in various ways, the importance of a particular node for the functionality of the overall network, based on its embedded position in the broader relationship network (known as the centrality of the node). Depending on the context, various centrality measures can be adopted to identify the key players of a given network. Table 6 in Appendix A presents the list of nodelevel metrics used in this study, and their implications within a SCN context.

2.3. Data-Driven Studies. Even though most theoretical modelling efforts of SCNs have focussed on variants of preferential attachment to generate network topologies for analysis [22], there have been some studies which have adopted a data-driven approach. For instance, Kim et al. [23] have undertaken a node- and network-level topological analysis, using three case studies of automotive supply networks (namely, Honda Accord, Acura CL/TL, and Daimler Chrysler Grand Cherokee) presented by Choi and Hong [24]. Although the SCNs used in this study are complete, the SCNs are small in size (largest network includes only 34 firms), which limits the observations of emergent network topological properties. Kito et al. [25] have constructed a SCN for Toyota using the data available within an online database operated by MarkLines Automotive Information Platform. By analysing the SCN topology, the authors have identified the tier structure of Toyota to be barrel-shaped, in contrast to the previously hypothesized pyramidal structure. Another fundamental observation reported in this study is that Toyota SCN topology was found to be not scale-free (even with finite-size effects taken into account). Although the dataset used in this study is sufficiently large (with 3109 firms), it is limited to only the top three tiers of the overall SCN.

More recently, using Bloomberg data, Brintrup et al. [26] and Orenstein [27] have undertaken topological analysis of various SCNs. Brintrup et al. [26] have studied the SCN of Airbus and have reported that this SCN displays assortative mixing and communities based on geographic locations of the firms. Orenstein [27] has undertaken topological analysis of retail and food industry SCNs by considering the suppliers within the top three tiers. The SCNs considered in this study were found to have scalefree topologies with degree exponents below 2. Although the dataset used in this study is sufficiently large and allows observation of temporal variations to the SCN topology, consideration of only a part of the SCN depth in terms of tiers has limited the generalizability of the results. It is noted that the key limitation in using the Bloomberg database, for constructing SCNs, is that the data are not exhaustive since the database only includes publicly listed firms. Therefore, the SCNs constructed using Bloomberg data may only provide a part of the full picture.

Although the above studies have provided a number of key insights about the topological structure of various SCNs, no study to date has systematically investigated a large collection of directed material flow SCNs in various industry sectors and compared the results against an undirected SCN of contractual (interfirm) relationships.

By considering a collection of twenty-six SCNs from the manufacturing industry, our study is able to investigate and establish the general topological properties of these SCNs. This effort will complement the large body of theoretical literature on modelling SCN topologies through various growth models, by revealing what specific topological characteristics are needed to be captured in an appropriate growth model. In addition, the correlation analysis presented in this study, between various node-level centrality measures and two exogenous factors (stage cost and stage time), can be powerful in demonstrating how the position of firms can influence the overall functionality of the SCN (and vice versa).

Finally, this study has used the reliable dataset provided in published work by Willems [14] in relation to the DMF-SCNs. The data collection procedure for the UCR-SCNs is detailed in Section 3.1.1. Our study offers distinct insights from previous studies because (i) it is based on a large collection of real-world SCNs belonging to the manufacturing sector, (ii) most of the SCNs are large (have a relatively high number of nodes) so that various emergent properties can be sufficiently demonstrated, (iii) we extract and compare the topological properties of SCNs where links represent either the directed material flows or undirected contractual relationships, (iv) our correlation analysis of node centrality measures with exogenous factors in DMF-SCNs provides insights into the impact of the position of firms in the dynamics of SCNs, and (v) we investigate the self-organization in all SCNs, which helps us identify nonrandom features of these systems. Thus, this study is unique on several counts from previous studies described above.

2.4. Generation of Null Models for Hypothesis Testing. An important question when testing hypothesis related to network topologies is whether the degree distribution on its own is sufficient to describe the structure of a network, i.e., whether the topological features observed in the network are explained by the ensembles of networks generated by its degree distribution while preserving the degree vector. In this regard, degree-preserving randomisation (DPR) plays an important role in generation of null models.

DPR involves rewiring the original network, to generate an ensemble of null models, while preserving the degree vector $[28,29]$. At each time step, the DRP process randomly picks two connected node pairs and switches their link 
targets. This switching is repeatedly applied to the entire network until each link is rewired at least once. The resulting network represents a null model where each node still has the same degree, yet the paths through the network have been randomised. Comparison of properties of a given network, with the properties of an ensemble of networks generated by DPR, allows one to identify if the properties observed in the real network are unique and meaningful or whether they are common to all networks with that degree sequence [30].

In this study, we use DPR to investigate the presence of self-organized topological features in the undirected contractual relationships SCN. For the DMF-SCNs, we introduce a novel DPR procedure to generate null models by preserving both the degree of nodes and the numbers of links present between adjacent tiers.

2.5. Robustness of SCNs. Past studies have gained insights into the robustness of SCNs by using (1) analytical measurements available in network science, such as network centralisation, percolation threshold, and assortativity, or (2) simulations to investigate how various network-level metrics are affected when nodes are sequentially removed, either randomly (known as random failures) or based on degree (known as targeted attacks) $[3,8,11,12]$.

While the above methods may be suitable for obtaining high-level insights into the robustness structure of UCRSCNs, a more specific method needs to be formulated for assessing the robustness of tiered DMF-SCNs. In particular, this method should consider both the topological structure of the network and its ability to meet the consumer demands. In this regard, we introduce a simple and intuitive method to investigate the robustness of DMF-SCNs, considering the demand at the retailer nodes.

\section{Data Sources, Structure, and Limitations}

\subsection{Data Sources and Structure}

3.1.1. Undirected Contractual Relationship SCN. The undirected contractual relationship SCN consists of customersupplier contractual linkages in the Indian automotive industry. The network is constructed from the citations of the autocomponent firms about the firms they offer their products and services to (in this regard, a firm could be a supplier to one firm and a customer of another firm). The list of firms is taken from the Automotive Component Manufacturers Association (ACMA) of India annual publication "Buyers Guide." The data corresponds to the year 2001-2002.

The basic topological analysis of this data (excluding the robustness analysis and self-organization analysis presented in this paper) was presented in Parhi [13]. Although this data was originally created from a 2002 directory, it was compared with recent records as a part of a study titled "Dynamics of Distribution and Diffusion of New Technology" [31] and the authors demonstrated that there is no qualitative difference in the industry structure compared to the 2002 dataset. In fact, the actors and their topography in the supply chain in the Indian Automotive Industry has remained more or less the same over the years (note that this dataset only includes the organized sector of the Indian automotive industry), where the only change has occurred with regard to the dynamics of the interactions, thanks to the fast integration of the information system in a highly integrated world.

3.1.2. Directed Material Flow SCNs. Willems [14] provides a dataset of real-world multiechelon supply chains, used for inventory optimization purposes. The overall dataset includes a total of 38 multiechelon supply chains, from various industries. The chains described in this paper comprise actual supply chain maps created by either company analysts or consultants. Since these maps have been implemented in practice, they demonstrate how users have modelled actual supply chains.

The above-mentioned dataset includes the following key information:

(1) The industry sector of each supply chain network

(2) For each supply chain network:

(i) The stages (nodes) representing each firm involved

(ii) The arcs (links) representing precedence relationship between stages

(3) For each stage:

(i) Its classification and tier based on its function within the overall supply chain

(ii) The direct cost added at the stage (stage cost)

(iii) The average processing time at the stage (stage time)

(iv) The average daily demand at each retailer stage

Note that in the network models developed in this paper, we denote stages (i.e., firms) as nodes and the arcs between stages (which represent the precedence relationships between firms) as directed links.

From the original dataset, networks with more than 100 firms (i.e., nodes) were selected for our analysis, and there were twenty-eight such large networks. Smaller networks were omitted in this analysis since they do not offer any interesting insights into emergence of various complex topological features. Then, using the industry sector information, these SCNs were categorised into six main groups as illustrated in Table 1. As can be seen, the set of SCNs considered by us vary in size (with a minimum of 108 to a maximum of 2025 nodes).

\subsection{Limitations of the Datasets}

3.2.1. Undirected Contractual Relationship SCN. The SCN data for the Indian automotive industry covers the organized sector of the industry, as there is no comprehensive database on the unorganized segment. The list of customers includes 
TABLE 1: Classification of directed material flow SCNs considered in the study.

\begin{tabular}{|c|c|c|c|c|c|}
\hline Reference & Industry group & Subindustry classification & Number of tiers & Total nodes & Total links \\
\hline 1 & \multirow{2}{*}{ Aircraft engines and engine parts } & N/A & 6 & 468 & 605 \\
\hline 2 & & $\mathrm{~N} / \mathrm{A}$ & 4 & 2025 & 16,225 \\
\hline 3 & \multirow{2}{*}{$\begin{array}{l}\text { Arrangement of transportation } \\
\text { of freight and cargo }\end{array}$} & $\mathrm{N} / \mathrm{A}$ & 4 & 116 & 119 \\
\hline 4 & & $\mathrm{~N} / \mathrm{A}$ & 5 & 626 & 632 \\
\hline 5 & \multirow{8}{*}{ Chemical } & $\begin{array}{l}\text { Soap and other detergents, } \\
\text { except specialty cleaners }\end{array}$ & 4 & 133 & 164 \\
\hline 6 & & $\begin{array}{l}\text { Perfumes, cosmetics, and } \\
\text { other toilet preparations }\end{array}$ & 7 & 186 & 359 \\
\hline 7 & & Pharmaceutical preparations & 9 & 253 & 253 \\
\hline 8 & & $\begin{array}{l}\text { Paints, varnishes, lacquers, } \\
\text { enamels, and allied products }\end{array}$ & 3 & 271 & 524 \\
\hline 9 & & Primary batteries, dry and wet & 5 & 617 & 753 \\
\hline 10 & & $\begin{array}{l}\text { Perfumes, cosmetics, and } \\
\text { other toilet preparations }\end{array}$ & 5 & 844 & 1685 \\
\hline 11 & & $\begin{array}{l}\text { Perfumes, cosmetics, and } \\
\text { other toilet preparations }\end{array}$ & 8 & 976 & 1009 \\
\hline 12 & & Industrial organic chemicals & 4 & 1479 & 2069 \\
\hline 13 & \multirow{6}{*}{ Computer-related } & Semiconductors and related devices & 2 & 108 & 452 \\
\hline 14 & & Computer peripheral equipment & 5 & 152 & 211 \\
\hline 15 & & Computer peripheral equipment & 8 & 154 & 224 \\
\hline 16 & & Computer peripheral equipment & 9 & 156 & 263 \\
\hline 17 & & Computer peripheral equipment & 10 & 156 & 169 \\
\hline 18 & & Computer storage devices & 8 & 577 & 2262 \\
\hline 19 & \multirow{5}{*}{ Electrical } & $\begin{array}{c}\text { Electromedical and } \\
\text { electrotherapeutic apparatus }\end{array}$ & 7 & 145 & 224 \\
\hline 20 & & Power-driven handtools & 6 & 334 & 1245 \\
\hline 21 & & $\begin{array}{c}\text { Electromedical and } \\
\text { electrotherapeutic apparatus }\end{array}$ & 5 & 482 & 941 \\
\hline 22 & & Telephone and telegraph apparatus & 3 & 1206 & 4063 \\
\hline 23 & & $\begin{array}{c}\text { Electromedical and } \\
\text { electrotherapeutic apparatus }\end{array}$ & 6 & 1386 & 1857 \\
\hline 24 & \multirow{3}{*}{ Farm machinery and equipment } & $\mathrm{N} / \mathrm{A}$ & 3 & 409 & 853 \\
\hline 25 & & N/A & 4 & 706 & 908 \\
\hline 26 & & $\mathrm{~N} / \mathrm{A}$ & 4 & 1451 & 4812 \\
\hline
\end{tabular}

only the firms that have been self-reported by the autocomponent firms as their principal customers.

3.2.2. Directed Material Flow SCNs. A key limitation of the available dataset is the lack of information in relation to the geographical locations of individual firms. This information was not provided in the original dataset in Willems [14] due to confidentiality reasons. Unlike the virtual networks (such as WWW or social networks), the SCN structure is largely influenced by geographical aspects (since the congregation or dispersion of the suppliers depends on the raw material distribution over various geographic regions). Therefore, if geographic location information was available, in-depth conclusions could have been made about various observed structural features of the SCNs.

In addition, this study is unable to investigate the dynamic nature of the SCNs since the dataset does not provide any information pertaining to temporal changes in the SCN topology. Lastly, the relationship strength between firms is not captured in the dataset in terms of the amount material flow. Although specific production capabilities of firms within each tier are known, no information is available in relation to how much each upstream firm supplies to the downstream firms.

Nevertheless, the size of the dataset, both in terms of the number of networks available and the size of each network, as well as the cost and time data associated with nodes make this a very attractive dataset to study.

\section{Data Analysis Methodology and Results}

4.1. Topological Results. Using each dataset, we constructed SCNs, where the nodes represent the individual firms and the links represent either the undirected contractual 


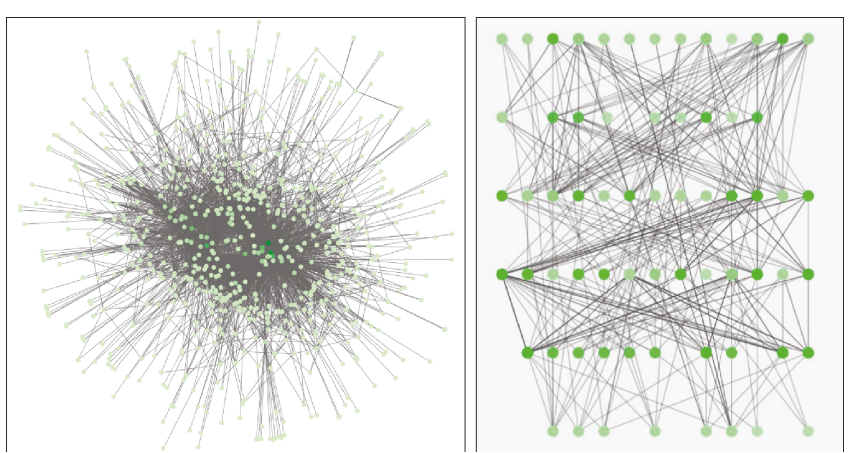

FIgURE 1: Visualisation of (1) the UCR-SCN (left), and (2) the tiered DMF-SCN in reference 6 (right). The shade of green is set proportional to the node degree.

TABle 2: Basic topological features of the undirected contractual relationship SCN.

\begin{tabular}{lc}
\hline Topological feature & Value \\
\hline No. of nodes & 687 \\
No. of links & 3283 \\
Average degree & 9.557 \\
Network centralisation & 0.296 \\
Network density & 0.014 \\
Network heterogeneity & 1.815 \\
Network diameter & 7 \\
Characteristic path length & 3.07 \\
Clustering coefficient & 0.065 \\
Assortativity (degree) & -0.292 \\
$\gamma\left(R^{2}\right.$ for power law) & $2.31(0.95)$ \\
\hline
\end{tabular}

relationships or the directed material flows between firms (see Figure 1). Cytoscape software and JAVA programming language were used to visualise and analyse the SCNs. The results are presented in the following subsections.

4.1.1. Undirected Contractual Relationship SCN. Table 2 presents the network-level topological features of the UCR-SCN of Indian automobile manufacturers.

4.1.2. Directed Material Flow SCNs. Table 3 presents the topological features of the 26 directed material flow SCNs. The three key observations that can be made from the table are that (i) the degree distributions of the majority of SCNs indicate good $R^{2}$ correlations against the power law fit, and most of them have degree exponents in the range of 1 to 3 , (ii) most $\mathrm{SCNs}$ are disassortative, in terms of degree as well as stage cost and stage time, and (iii) none of the SCNs indicated clustering (not presented in the table), due to the links only being present between functional tiers. These observations have important implications, which are discussed in more detail in Section 5.

In addition, for this dataset, we have investigated the node-level centrality metrics. Since node-level metrics themselves provide information about individual nodes rather than networks as a whole, here we choose to primarily study correlations between them and other node-level attributes available in the dataset. In particular, we studied the correlation coefficients between the centrality metrics for each node (namely, in- and out-degree centrality, betweenness centrality, and closeness centrality) and its corresponding (1) stage cost and (2) stage time. The correlation plots are presented in Appendix B. The results of this assessment are discussed in detail in Section 5.

\subsection{Identifying Self-Organized Topological Features Using} Degree-Preserving Randomisation. DPR can help establish whether or not the observed topological property in a network is simply an artefact of the network's inherent structural properties or a property unique to the nodes. Comparison of the original betweenness and closeness centrality distributions with the average distributions of the same metrics, for an ensemble of randomised (through DPR) networks, can reveal whether the centrality distributions observed in the original network are structural or not. In particular, if the original (i.e., observed) and randomised trends are identical, then the centrality distributions observed in the original network are purely structural, i.e., they can entirely be explained by the degree distribution without attribution to any other external mechanism. However, if the original centrality distribution diverges from the average trend obtained for the randomised ensembles, there is an underlying mechanism which induces this deviation in the original network [17].

Although the divergence between the observed centrality distribution and the randomised ensemble average is generally found through visual inspection of the plots $[17,32]$, in this study we have used the KolmogorovSmirnov test (KS test) to establish any statistically significant deviations. The KS test is a powerful statistical test that allows one to compare two distributions (the null hypothesis of the test is that there exists no difference between the two distributions). Importantly, the KS test does not make any assumption about the underlying distribution of the data (i.e., it is a nonparametric and distribution-free test statistic), thus allowing comparisons between arbitrary distributions.

4.2.1. Undirected Contractual Relationship SCN. For the UCR-SCN, we applied DPR where, at each time step, two connected node pairs are picked and their links targets switched. This switching is repeatedly applied to the entire network until every link is rewired at least once (without allowing the creation of self-loops or multilinks between node pairs). The resulting network represents a null model where each node still has the same degree, yet the paths through the network have been randomised. Using this process, we generated 1000 randomised networks. We have then compared the betweenness and closeness centrality distributions of the original network with the average betweenness and closeness values obtained for the 1000 randomised networks (see Figure 2). 


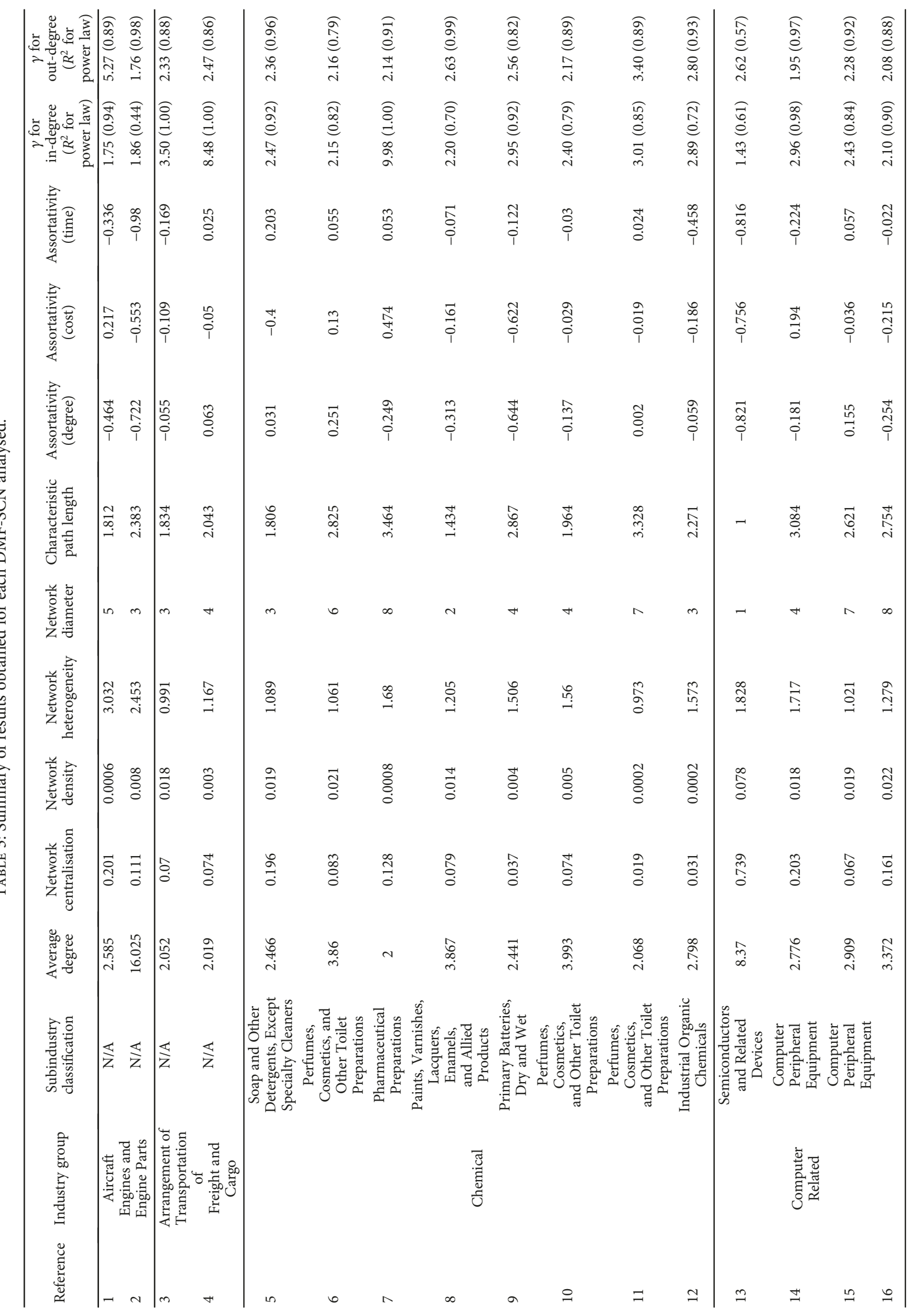




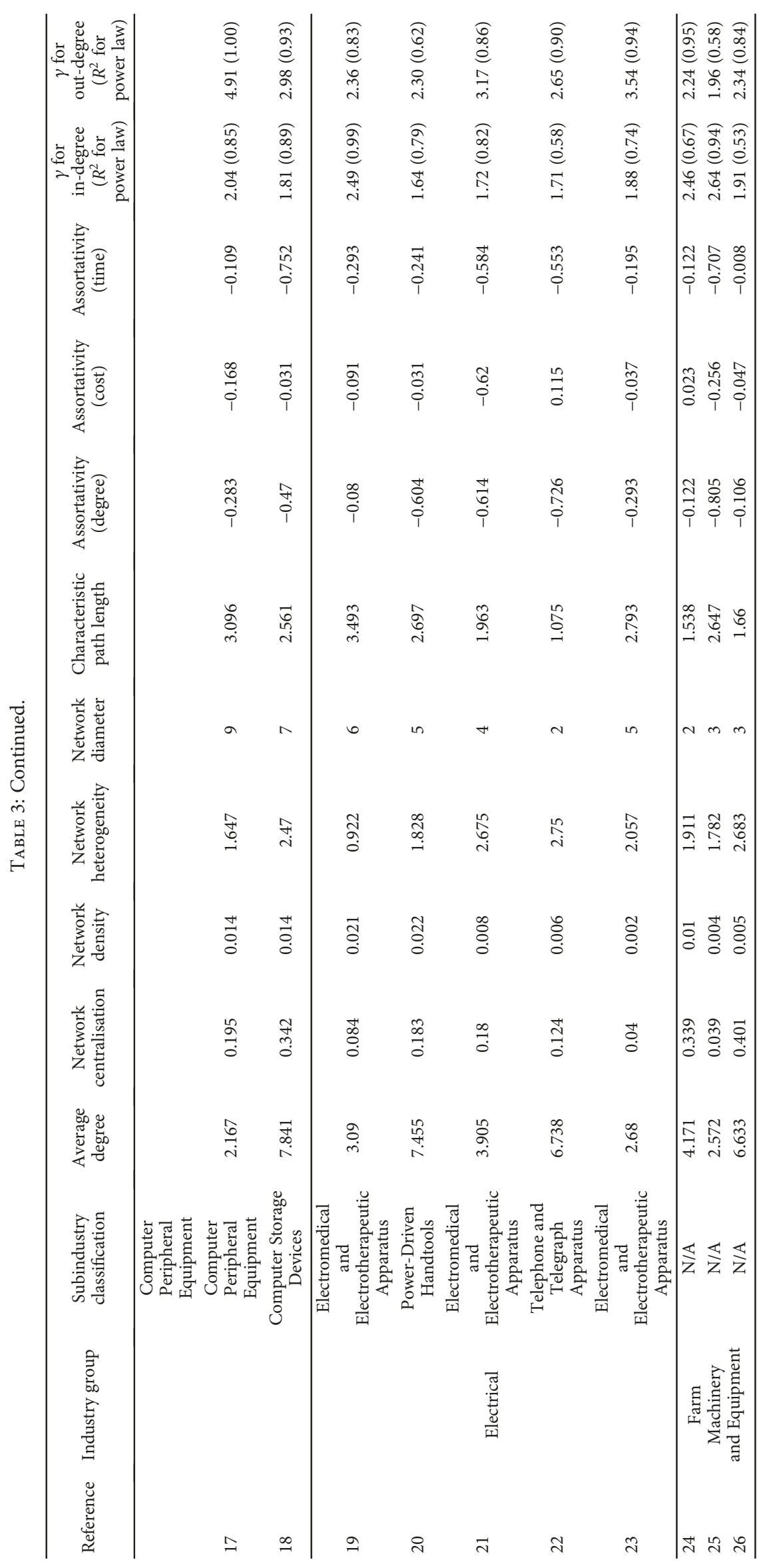




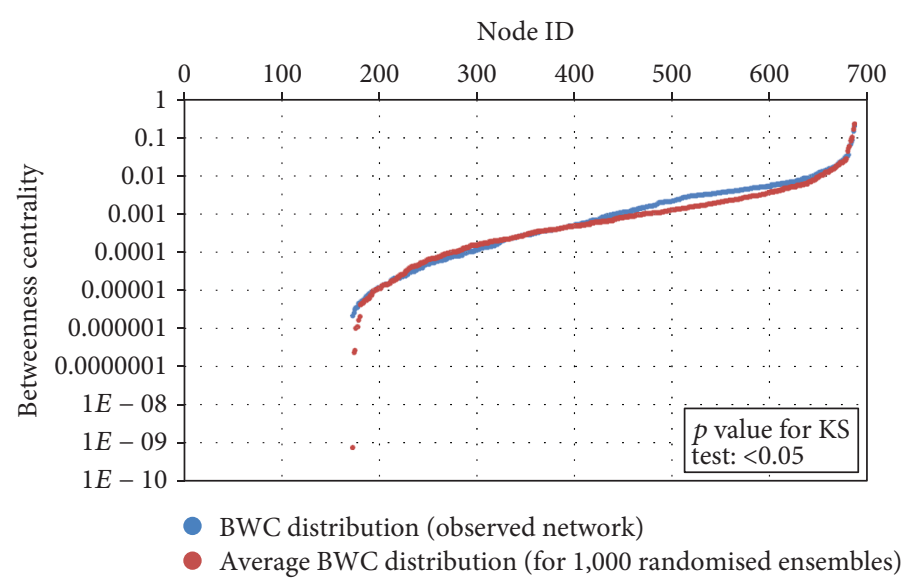

(a)

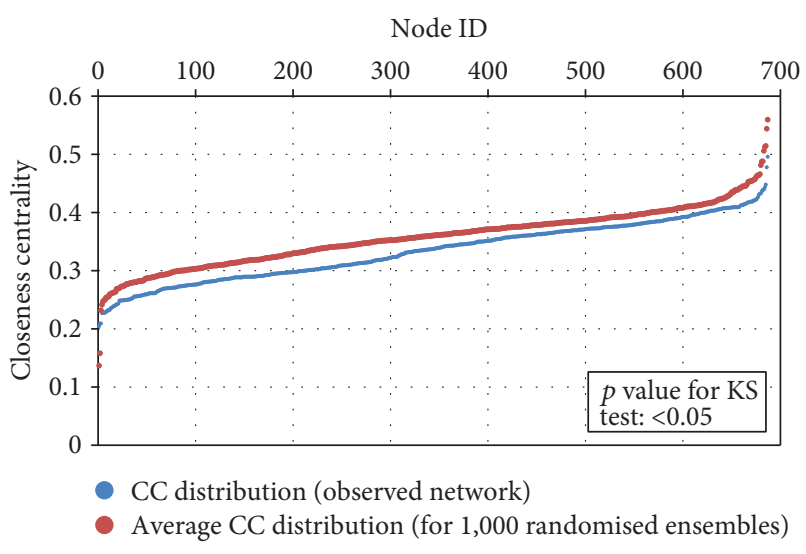

(b)

FIGURE 2: (a) Betweenness centrality distribution of the observed SCN against the average betweenness centrality distribution obtained from 1000 randomised ensembles. (b) Closeness centrality distribution of the observed SCN against the average closeness centrality distribution obtained from 1000 randomised ensembles.

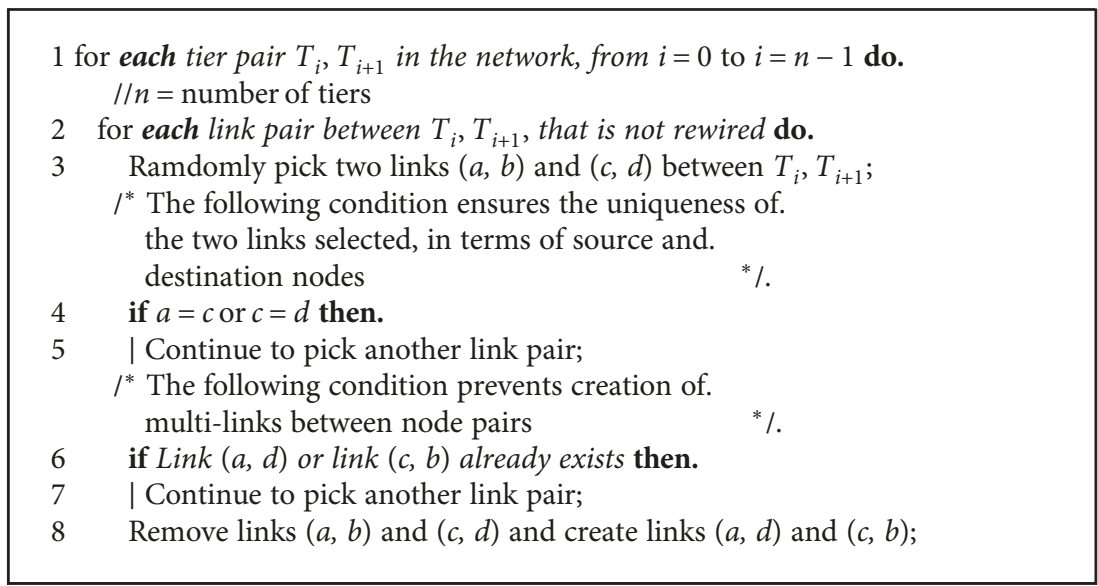

Algorithm 1: Modified degree-preserving randomizing algorithm for tiered networks.

4.2.2. Directed Material Flow SCN. For the DMF-SCNs, we have developed a modified DPR procedure, which we refer to as the tier constrained DPR (TC-DPR). At each time step, this process picks a pair of links which lie across the same two tiers and swaps their target nodes. In particular, the following algorithm has been used.

The above process is repeatedly applied to the original network until every link is rewired at least once. By choosing link pairs between the same two tiers and swapping their targets, in addition to preserving the degree of nodes, we also preserve the number of intertier links. Therefore, the resulting network is a null model whose degree distribution and tier structure are identical to the original SCN.

Using the TC-DPR process, we generated 1000 randomised networks. We have then compared the betweenness and closeness centrality distributions of the original network with the average betweenness and closeness values obtained for the 1000 randomised network ensembles. The $p$ values obtained for the KS test for each SCN is presented in Table 4. Figures 3-6 illustrate some scenarios where self-organization in terms of betweenness and closeness was identified.

4.3. Robustness Analysis. In this section, we investigate the robustness of the UCR-SCN. Subsequently, a simple and intuitive methodology for assessing the robustness of tiered DMF-SCNs with demand considerations is introduced.

4.3.1. Undirected SCN. Since the UCR-SCN dataset does not include any node attributes, we resort to the generic topological robustness analysis technique commonly employed in network science literature. In particular, we remove nodes, either randomly (to simulate random failures) or sequentially based on their degree (to simulate targeted attacks). In each iteration, we measure the size of the largest connected component (LCC) of the network. As nodes are successively removed, the overall network disintegrates into numerous subnetworks. The number of nodes in the LCC of 
TABLE 4: $p$ values obtained for the KS test for observed centrality distributions and the average centrality distributions obtained for 1000 randomised ensembles, for various SCNs.

\begin{tabular}{|c|c|c|c|c|}
\hline Reference & Industry group & Subindustry classification & $\begin{array}{c}\text { Observed BWC } \\
\text { against randomised } \\
\text { ensemble averages } \\
(p \text { value for the KS test) }\end{array}$ & $\begin{array}{c}\text { Observed CC } \\
\text { against randomised } \\
\text { ensemble averages } \\
\text { ( } p \text { value for the KS test) }\end{array}$ \\
\hline 1 & \multirow{2}{*}{ Aircraft engines and engine parts } & N/A & $>0.1$ & $\leq 0.05$ \\
\hline 2 & & N/A & $\leq 0.05$ & $>0.1$ \\
\hline 3 & \multirow{2}{*}{$\begin{array}{l}\text { Arrangement of transportation } \\
\text { of freight and cargo }\end{array}$} & $\mathrm{N} / \mathrm{A}$ & $\leq 0.05$ & $>0.1$ \\
\hline 4 & & N/A & $\leq 0.05$ & $>0.1$ \\
\hline 5 & \multirow{8}{*}{ Chemical } & $\begin{array}{l}\text { Soap and other detergents, } \\
\text { except specialty cleaners }\end{array}$ & $\leq 0.05$ & $>0.1$ \\
\hline 6 & & $\begin{array}{l}\text { Perfumes, cosmetics, and } \\
\text { other toilet preparations }\end{array}$ & $>0.1$ & $>0.1$ \\
\hline 7 & & Pharmaceutical preparations & $\leq 0.05$ & $\leq 0.05$ \\
\hline 8 & & $\begin{array}{l}\text { Paints, varnishes, lacquers, } \\
\text { enamels, and allied products }\end{array}$ & $>0.1$ & $\leq 0.05$ \\
\hline 9 & & Primary batteries, dry and wet & $>0.1$ & $>0.1$ \\
\hline 10 & & $\begin{array}{l}\text { Perfumes, cosmetics, and } \\
\text { other toilet preparations }\end{array}$ & $>0.1$ & $>0.1$ \\
\hline 11 & & $\begin{array}{l}\text { Perfumes, cosmetics, and } \\
\text { other toilet preparations }\end{array}$ & $\leq 0.05$ & $\leq 0.05$ \\
\hline 12 & & Industrial organic chemicals & $\leq 0.05$ & $>0.1$ \\
\hline 13 & \multirow{6}{*}{ Computer-related } & Semiconductors and related devices & $>0.1$ & $>0.1$ \\
\hline 14 & & Computer peripheral equipment & $\leq 0.05$ & $>0.1$ \\
\hline 15 & & Computer peripheral equipment & $>0.1$ & $\leq 0.05$ \\
\hline 16 & & Computer peripheral equipment & $>0.1$ & $\leq 0.05$ \\
\hline 17 & & Computer peripheral equipment & $>0.1$ & $>0.1$ \\
\hline 18 & & Computer storage devices & $>0.1$ & $\leq 0.05$ \\
\hline 19 & \multirow{5}{*}{ Electrical } & $\begin{array}{c}\text { Electromedical and } \\
\text { electrotherapeutic apparatus }\end{array}$ & $0.1-0.05$ & $>0.1$ \\
\hline 20 & & Power-driven handtools & $>0.1$ & $\leq 0.05$ \\
\hline 21 & & $\begin{array}{c}\text { Electromedical and } \\
\text { electrotherapeutic apparatus }\end{array}$ & $>0.1$ & $>0.1$ \\
\hline 22 & & Telephone and telegraph apparatus & $>0.1$ & $>0.1$ \\
\hline 23 & & $\begin{array}{c}\text { Electromedical and } \\
\text { electrotherapeutic apparatus }\end{array}$ & $\leq 0.05$ & $\leq 0.05$ \\
\hline 24 & \multirow{3}{*}{ Farm machinery and equipment } & N/A & $\leq 0.05$ & $>0.1$ \\
\hline 25 & & $\mathrm{~N} / \mathrm{A}$ & $>0.1$ & $>0.1$ \\
\hline 26 & & $\mathrm{~N} / \mathrm{A}$ & $\leq 0.05$ & $\leq 0.05$ \\
\hline
\end{tabular}

the fragmented network therefore provides insights into its structural integrity in terms of overall connectivity.

Figure 7 illustrates the topological robustness assessment results. The size of the LCC has been plotted against the percentage of removed nodes, under random and targeted node removal scenarios. Note that for the random node removal scenario, the result has been obtained by averaging 100 runs of the simulation.

4.3.2. Directed SCNs. Unlike the UCR-SCN, the Willems [14] dataset for DMF-SCNs includes average daily demand levels at retailer nodes. Therefore, in order to more accurately assess the robustness of the DMF-SCNs, we develop a simple and intuitive methodology. In particular, we establish the robustness of each network as a function of lost average daily demand, as firms are sequentially removed. In developing the robustness assessment method, we assume that the firms within the same tier have substitutable capabilities with no capacity restrictions, i.e., the supplies lost due to failure of one firm can be fully replenished by another firm in the same tier.

For instance, consider the hypothetical DMF-SCN scenario presented in the Figure 8. In order for retailer B to satisfy its average daily demand, it relies on three supply chains, namely, (1) B-F-J-N, (2) B-F-J-M, and (3) B-F-I-M. Now consider a scenario where the manufacturing firm $\mathrm{J}$ 


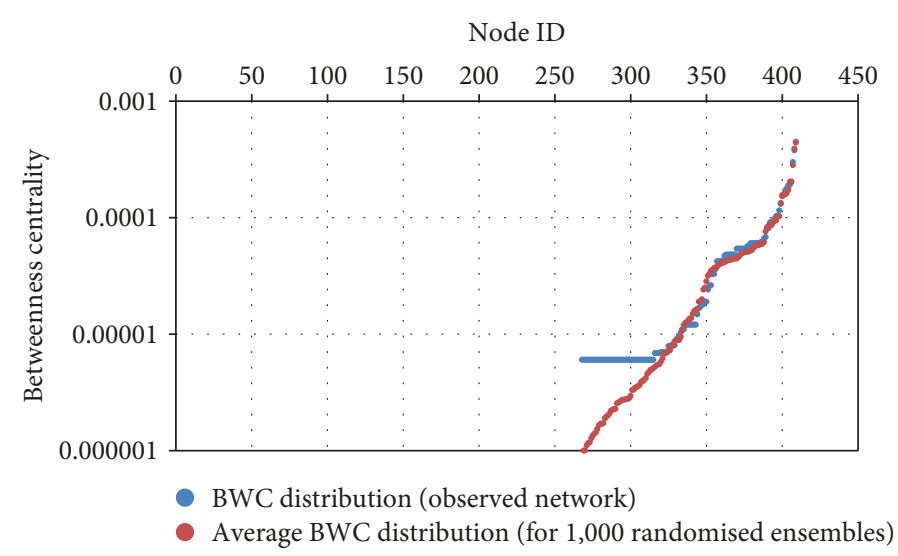

FIGURE 3: Betweenness centrality distribution of the observed SCN (ref \#: 24-Farm Machinery and Equipment) against the average betweenness centrality distribution obtained from 1000 randomised ensembles.

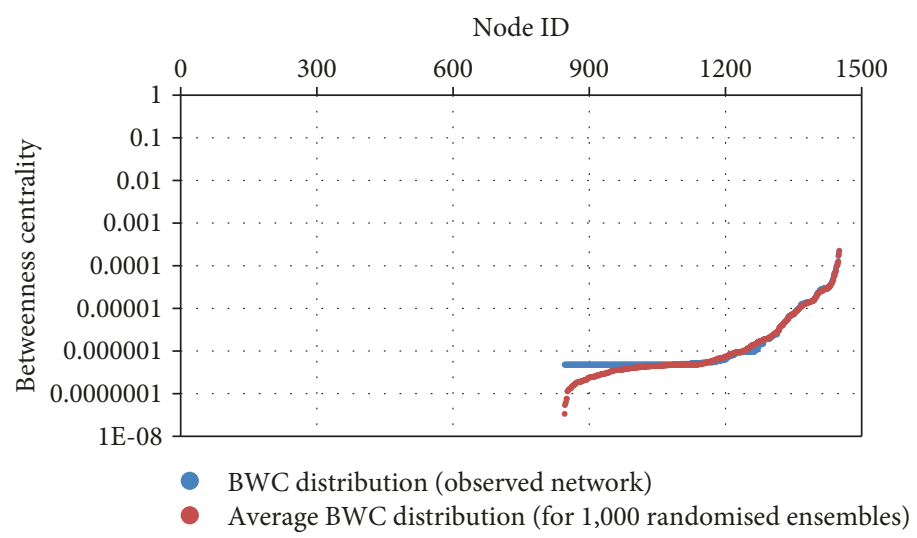

FIGURE 4: Betweenness centrality distribution of the observed SCN (ref \#: 26-Farm Machinery and Equipment) against the average betweenness centrality distribution obtained from 1000 randomised ensembles.

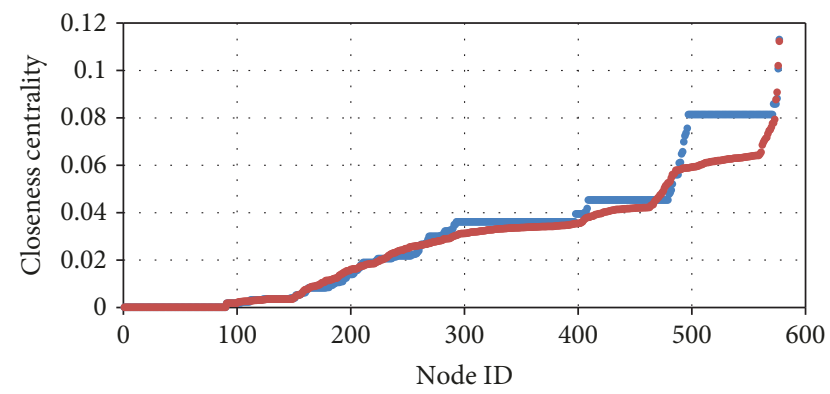

CC distribution (observed network)

Average CC distribution (for 1,000 randomised ensembles)

FIGURE 5: Closeness centrality distribution of the observed SCN (ref \#: 18-Computer Storage Devices) against the average closeness centrality distribution obtained from 1000 randomised ensembles.

is removed from the network-in this case, retailer B will lose two out of the three supply chains it relies on to satisfy its average daily demand. However, retailer B will still be able to satisfy its average demand through the supply chain B-F-I-M, which is not affected by removal of firm J. Therefore, as long as the retailer node has access to an upstream most supplier through a complete supply chain, it will be able to satisfy its average daily demand. However, when firm J is removed, retailer D will not be able to satisfy its average daily demand-since both supply chains for D (D-G-J-M and D-G-J-N) are reliant on firm J. In this regard, it is important to note that removal of any retailer node implies that it will not be able to satisfy its respective average daily demand.

Based on the above idea, we develop a robustness metric termed "Robustness Score" which is defined as follows:

$$
\text { Robustness Score }=\sum_{r=1}^{R} \delta_{r} D_{r} \text {, }
$$

where $R$ is the set of retailers in the SCN and $D_{r}$ is the average daily demand at retailer $r . \delta_{r}$ captures the availability of paths to the upstream most suppliers at each retailer and is 1 if there exists at least one path connecting the retailer to an upstream most supplier and 0 otherwise. 


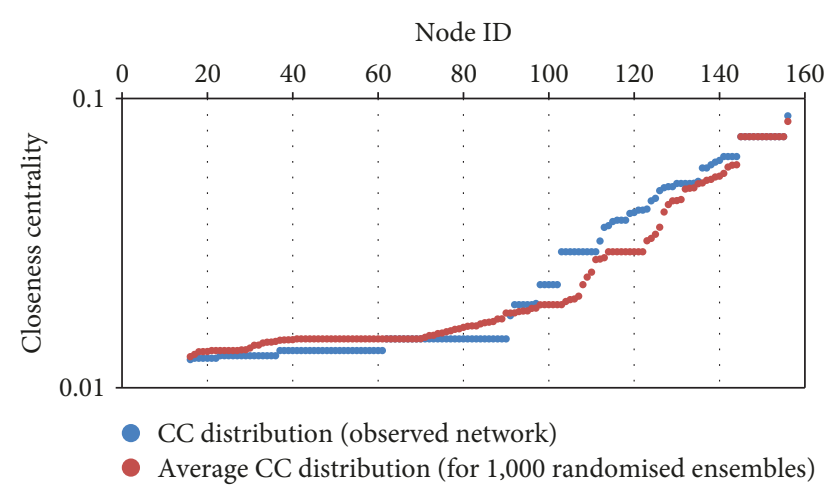

FIGURE 6: Closeness centrality distribution of the observed SCN (ref \#: 16-Electromedical and Electrotherapeutic Apparatus) against the average closeness centrality distribution obtained from 1000 randomised ensembles.

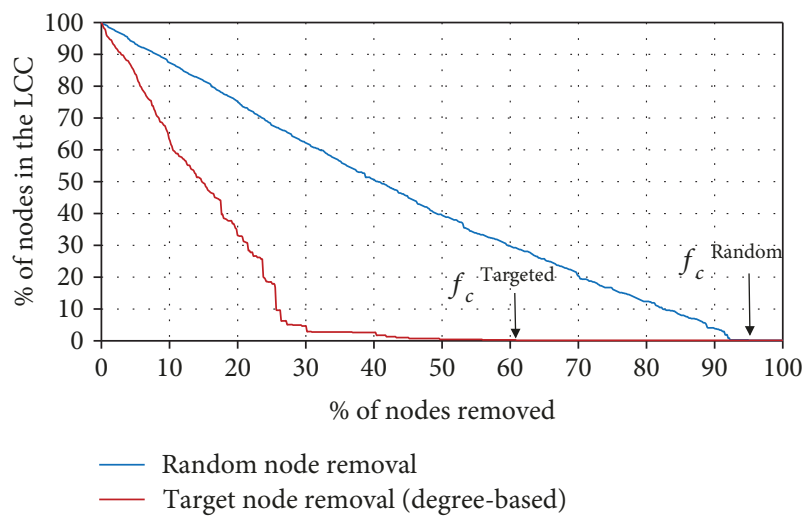

Figure 7: Percentage of nodes in the largest connected component under random and targeted (degree-based) node removal.

We applied the above idea of Robustness Score to the SCN ref 20 (power-driven handtools), where nodes were removed randomly and sequentially based on their degree (i.e., targeted attacks). For each scenario, after the removal of each node, the drop in Robustness Score was recorded to generate a profile. This result is presented in Figure 9.

\section{Discussion}

5.1. Topological Structure of the SCNs. The degree distributions for the majority of the DMF-SCNs indicate a good fit with power law. In particular, 14 out of the 26 networks analysed display $80 \%$ or higher $R^{2}$ correlation with a power-law fit for both their in- and out-degree distributions The degree exponents of all DMF-SCNs were generally found to lie in the range of 1 to 3: note that $\gamma=2$ is the boundary between hub and spoke $(\gamma<2)$ and scale-free $(2<\gamma<3)$ network topologies. Similar findings were observed for the UCR-SCN, which displayed a $95 \% R^{2}$ correlation with a power-law fit for its degree distribution with a degree exponent of 2.31.

These results are in good agreement with the empirical findings on SCNs reported in recent data-driven studies
$[26,27,33,34]$, which indicate the topologies of SCNs tend to have degree distributions which can be satisfactorily modelled by power law, with degree exponent $\approx 2[22]$.

Although a number of past theoretical studies have relied on the Barabási-Albert (BA) model to generate topologies representative of SCNs $[3,10,12]$, based on our results, it is evident that the BA model cannot sufficiently explain the intricacies of real-world SCN topologies. In particular, the BA model generates network topologies with $\gamma=3$ [35], while the real-world SCNs indicate $\gamma$ in the range of 1 to 3 . Also, the assortative (or disassortative) mixing observed in real SCNs is not a feature of networks generated by the BA model, as shown analytically (in the limit of large network size) by Newman [36]. Finally, the BA model cannot generate networks with pronounced community structure which has been observed in real SCNs, since all nodes in the network belong to a single weakly connected component [37].

Indeed, a range of network growth models are available in extant literature, and broadly speaking, they can be categorised as either evolving models or generative models [17]. The evolving models are aimed at capturing the microscopic mechanisms underlying the temporal evolution of a network topology. In this regard, the BA model can be regarded as an evolving model. In contrast, generative models can be used to generate a snapshot of a network topology. Since the SCNs may have evolved based on various nongeneralizable principles, rather than attempting to understand and model the underlying growth mechanism through an evolving network growth model, it would be beneficial to simply mimic the observed topologies from data-driven studies using a generative network model.

In this regard, fitness-based generative models have recently gained prominence in theoretical research ([38-40]; Smolyarenko, 2014; [41]). In fitness-based models, the fitness distribution and the connection rules are given by a priori arbitrary functions, which enable a considerable amount of tuning (Smolyarenko, 2014). Indeed, this tunability makes such models a useful and practical modelling tool. For example, Ghadge et al. (2010) have proposed a purely statistical method for generating a range of network topologies by randomly allocating the nodes with fitness values sampled from a log-normal distribution. The propensity of each node to attract links is determined proportionally to its fitness. This method is referred to as the log-normal fitness attachment (LNFA), and it includes a tunable parameter $\sigma$ (the shape parameter of the log-normal distribution), which can be manipulated to generate a large spectrum of networks. At one extreme, when $\sigma$ is zero, all nodes have the same fitness and therefore at the time a new node joins the network, it chooses any existing node as a neighbour with equal probability, thus replicating the random graph model. On the other hand, when $\sigma$ is increased beyond a certain threshold, very few nodes will have very large levels of fitness while the overwhelming majority of nodes have extremely low levels of fitness. As a result, the majority of new connections will be made to a few nodes which have high levels of fitness. The resulting network therefore resembles a monopolistic/"winner-take-all" scenario, which can sometimes be observed in the real world. Between the above two extremes (random 


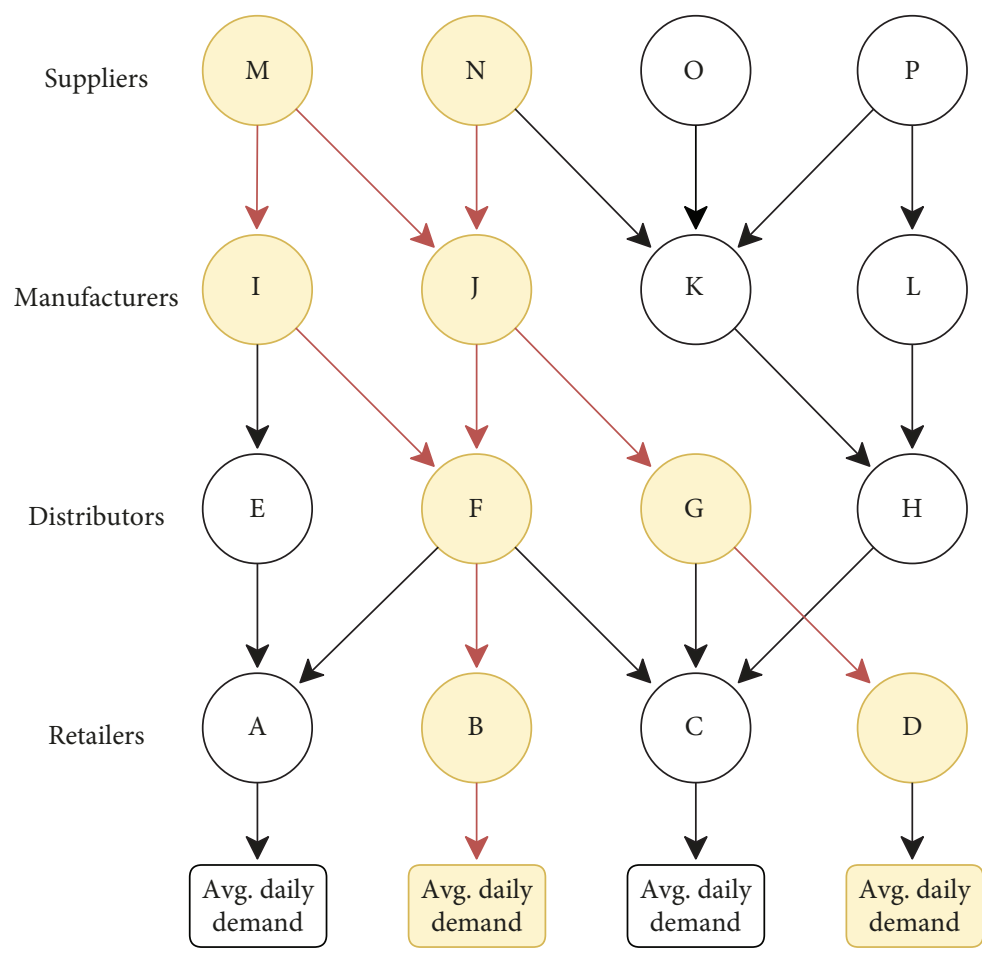

Figure 8: Path structure of a typical DMF-SCN.

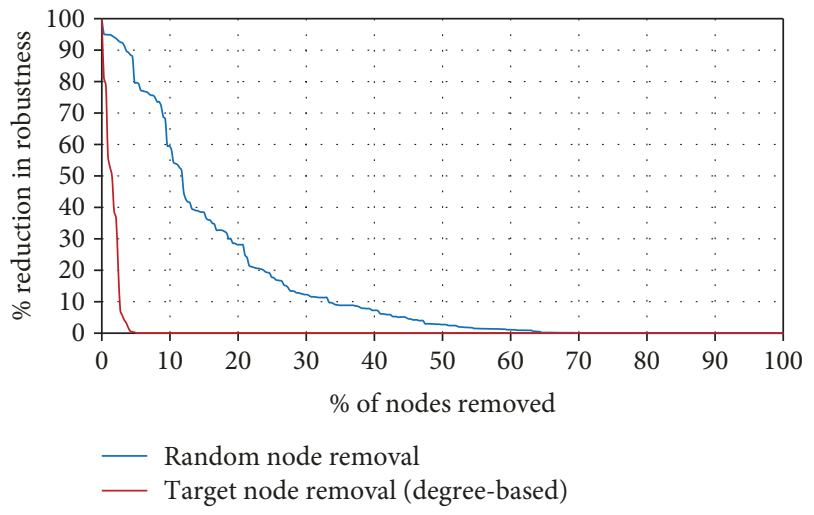

FIgURE 9: Effect of random and targeted (degree based) node removals on overall SCN robustness.

and monopolistic) lies a spectrum of power law networks which can closely represent many real SCNs (Ghadge et al., 2010). Nguyen and Tran [42] have illustrated that the LNFA model can indeed generate network topologies with $\gamma$ in the range of 2-3 (and beyond), which can realistically represent the observed SCN topologies. Recent work by Bell et al. [43] has shown that a risk-averse behaviour by SCN firms can lead to a fitness-based network growth.

It is also interesting to note that all the DMF-SCNs indicate relatively lower network centralisation values llustrating the largely distributed and decentralised nature of modern SCNs. This lower centralisation could also be due to the recent supply chain practice known as modular assembly, where manufacturers obtain pre-assembled modules from a reduced base of suppliers (such as through intermediate subassemblers), in contrast to the traditional approach in which individual components are procured and assembled by the manufacturer [44]. In general, the SCNs which involve more complex and specialised manufacturing and assembly processes (such as aircraft parts, computer equipment and, electrical and farm machinery) were found to be more centralised than the other SCNs such as cargo and chemicals (which is also indicated by its low level of heterogeneity).

The network centralisation for the UCR-SCN was found to be higher than the average network centralisation for the DMF-SCN dataset. This could be due to the inherent differences between the two types of the SCNs, i.e., the contractual relationships are generally centralised through a leader firm whereas the flow networks generally link firms who wish to exchange material/goods.

In terms of degree assortativity, majority of the DMFSCNs (21 out of 26) were found to be slightly or strongly disassortative, where the highly connected hubs tend to avoid each other, instead linking to lower-degree nodes. As a result, the network structure of these SCNs tends to display a huband-spoke character (as opposed to core periphery structure observed in assortative networks). Some SCNs in industries such as "Perfumes, Cosmetics, and Other Toilet Preparations" and "Semiconductors and Related Devices" were strongly disassortative. We found no SCN which was strongly assortative. Additionally, the UCR-SCN was also found to be disassortative, in terms of node degree. This type of disassortative mixing has been observed commonly in economic systems [17] where trade typically takes place 
between individuals or organizations of different skills and specialities.

An unfavourable implication of the disassortativity observed in the SCNs (in terms of degree) is that since high degree nodes are less connected to one another, many paths between nodes in the network are dependent on high degree nodes. Therefore, failure of a high degree node in a disassortative network would have a relatively large impact on the overall connectedness of the network [29]. On the other hand, disassortative networks are generally resilient against cascading impacts arising from targeted attacks-since hub nodes are not connected with each other, the likelihood of disruption impacts cascading from one hub node to another is minimised [45].

Furthermore, the DMF-SCNs mostly displayed slight or strong disassortative tendencies in terms of stage-cost and stage-time attributes ( 20 out of $26 \mathrm{SCNs}$ were found to be disassortative in terms of stage cost and stage time). That is, firms which contribute high stage cost are on average more likely to be connected with firms that contribute low stage cost, and vice versa, and the same is true for stage time. No SCN that we studied displayed a strong assortative tendency in terms of these attributes. The strictly tiered structure of these SCNs could be responsible for this stage-cost and stage-time disassortativity as it separates the functional capabilities of firms which are linked with each other between tiers.

An important difference between the UCR-SCN and the DMF-SCNs was observed in their clustering properties. The clustering coefficient indicates the degree to which firms in a SCN tend to cluster together around a given firm. For example, it can indicate how various suppliers behave with respect to the final assembler at the global level [23]. Therefore, the higher the clustering coefficient, the more dependent suppliers are on each other for production [46]. The UCR-SCN indicated low levels of clustering while none of the DMF-SCNs indicated any clustering. It is noted that in the DMF-SCN dataset considered, no horizontal connections were observed (i.e., no connections between firms within the same functional tier). Due to this inherent structural limitation, which prohibits triadic closure, these SCNs do not indicate any clustering (therefore, clustering values are not reported in Table 2). This implies that the suppliers in the material flow networks are independent of each other (since they are likely to be competitors). While this structure may limit diffusion of knowledge through the SCN, it has favourable implications in terms of network robustness, since disruptions to one supplier is unlikely to impact another. Hearnshaw and Wilson (2010) note that SCNs with a low clustering coefficient are likely to experience a more opportunistic behaviour and less collaboration which may lead to system inefficiencies due to the difficulty in system-wide coordination. Therefore, SCN efficiency can be improved by creating new horizontal connections, such as through social relationships. Choi and $\mathrm{Wu}$ [47] report a real-world example of such a scenario where Honda has built clustering in their supply chain by directing and facilitating the relationships of its first-tier suppliers with some second-tier suppliers.
5.2. Correlation between Topological Centrality Metrics and Other Firm Attributes in Directed Material Flow SCNs. For the DMF-SCNs, node centrality correlation assessments were carried out against node attributes. In general, for the majority of SCNs (regardless of the industry), we observed the following:

(1) The degree of firms was found to correlate positively with the stage cost while no correlation was identified with the stage time. This implies that the firms with higher number of connections generally add higher levels of direct costs to the market price of the final product.

(i) The in-degree of firms was found to correlate positively with the stage cost and negatively with stage time. This was particularly evident for the SCNs in aircraft engine and electrical industries. It implies that in these SCNs, the firms with higher number of upstream suppliers tend to add higher levels of direct costs to the market price of the final product and they tend to have relatively lower average processing times (these are firms which play the role of assemblers, which bring together many components from various suppliers for assembly purposes).

(ii) With the exception of SCNs in aircraft engine and electrical industries, the out-degree of firms was found to correlate positively with the stage cost and the stage time, suggesting that the firms with high number of downstream customers (such as major distributors) tend to add higher levels of direct costs to the market price of the final product and they tend to have relatively higher average processing times. This was particularly evident for the two SCNs in the transportation of freight and cargo industry.

(2) The betweenness and closeness centralities of firms were found to correlate positively with their stage cost, which implies that those firms which are more involved in the relationships between other pairs of firms and those firms which are active information generators tend to add higher values to the final product.

(3) The SCNs in electrical, computer-related, farm machinery and equipment, and aircraft engine industries indicated negative correlations between the betweenness centrality and stage time. This implies that, in these SCNs, the firms which are involved in the relationships between two other firms tend to require lower average processing times. Such central firms generally play the role of assemblers or distributors, thus requiring less processing times.

The above insights demonstrate how, from a SCN point of view, the position of an individual firm with respect to the others can influence both strategy and behaviour [48]. 


\subsection{Self-Organized Features}

5.3.1. Undirected SCN. Comparison of the betweenness and closeness centrality distributions of the original network with the average betweenness and closeness distributions obtained for the 1000 randomised networks (shown in Figure 2) reveals that there exists a significant difference (KS test $p$ value is $<0.05$ ) between the respective centrality distributions in the observed SCN and the average for the naturally occurring scheme.

In terms of betweenness centrality, we observe that moderate betweenness firms were overrepresented in the observed SCN compared to the average of the randomised ensembles. Therefore, we can conclude that the UCR-SCN topologies are nonrandom and favour moderate betweenness firms. A similar result was reported by Becker et al. [32] who constructed a manufacturing system network model from real-world data (where nodes represent separate work stations and links represent material flows between work stations). By applying the DPR process to generate an ensemble of networks with the same degree distribution, they observed that work stations with a particularly high betweenness centrality are overrepresented in the manufacturing system studied. They concluded that the manufacturing system topology is therefore nonrandom and favours the existence of a few highly connected work stations. Betweenness of a firm in the context of a UCR-SCN indicates the extent to which it can intervene over interactions among other firms in the SCN by being a gatekeeper for relationships [23]. Indeed, this has specific advantages in a networked economy, since it enables the firms to acquire more market intelligence and control by playing the role of an intermediator. Therefore, it is reasonable to see firms self-organizing themselves in order to increase their betweenness in the SCN.

In relation to closeness centrality, we observed that all firms in the UCR-SCN included lower closeness centralities when compared with the average of the randomised ensembles. Closeness indicates the proximity of a given firm with respect to others in the network. Complex manufacturing industries, such as the automobile manufacturing sector indicated in the UCR-SCN, include longer supply and service chains which can place the firms peripherally and far away from each other (as indicated by the network diameter of 7 for the UCR-SCN, which is higher than the majority of the DMF-SCNs).

5.3.2. Directed SCNs. Our results indicate that only some DMF-SCNs include self-organized features in terms of betweenness and closeness. Those SCNs with a $p$ value $\leq 0.05$ for the KS test, as outlined in Table 4, indicate that there exists the significant difference between the centrality distributions of the observed SCN and the average of the randomised network ensembles. This implies that in these SCNs, there exists an external mechanism beyond the degree distribution, which has driven the firms to adopt various centrality levels. In general, as illustrated in Figures 3 and 4, comparison of observed betweenness profiles against the average of randomised ensembles reveals that firms with low betweenness are underrepresented in the observed DMF-SCNs. This is an interesting observation which complements the findings obtained for the UCR-SCNs which indicated the moderate betweenness firms to be overrepresented. This implies that in material flow SCNs, the firms need to maintain a certain level of betweenness to function (as evident from the plateau of low betweenness nodes in the profiles of observed SCNs presented in Figures 3 and 4).

In contrast, comparison of observed closeness profiles against the average of randomised ensembles reveals that firms with moderate closeness are overrepresented in the observed DMF-SCNs (Figures 5 and 6). While it is closely related to betweenness centrality, closeness is more relevant in situations where a firm acts as a generator of information rather than a mere mediator/gatekeeper. For example, due to various hindrances, the market demand information can easily be distorted when it flows from the downstream firms towards upstream firms. Such distortions can lead to undue deviation between production plans of manufacturers and supply plans of suppliers, leading to a phenomenon known as the bullwhip effect. Firms with high closeness centrality levels therefore play a major role in sharing the actual market demand information with upstream firms in the SCN, thus diminishing the adverse impacts arising from the bullwhip effect [9]. This could be a reason why the firms in the DMF-SCNs have self-organized to have higher than random levels of closeness in the moderate closeness regime.

\subsection{Robustness Character}

5.4.1. Undirected SCN. The point identified as $f_{c}$ in Figure 7 indicates the critical threshold at which the LCC disappears and the network is fully fragmented into individual nodes. As can be seen from this figure, the network breaks apart relatively rapidly when the nodes are removed sequentially by targeting higher degree ones first, compared with random node removals. It is evident that $f_{c}^{\text {Targeted }}$ occurs at $60.93 \%$ while $f_{c}^{\text {Random }}$ occurs at $95.04 \%$.

It is interesting to investigate how different the observed robustness (against random node removals) of the network at hand is, when compared against a randomly wired network of the same size (in terms of the number of nodes and links). This question can be answered using the Molloy-Reed criterion which identifies the presence of a LCC within a network (Reed, 1995). This is on the basis that for a network to have a LCC, most nodes that belong to it must be connected to at least two other nodes [17]. In particular, the Molloy-Reed criterion states that a network has a LCC if

$$
\kappa=\frac{\left\langle k^{2}\right\rangle}{\langle k\rangle}>2,
$$

where $\langle k\rangle$ and $\left\langle k^{2}\right\rangle$ are the first (mean) and second moment of the network's degree distribution, respectively.

Based on the above, networks with $\kappa<2$ lack a LCC and are composed of many disconnected clusters. Applying the insight provided by the Molloy-Reed criterion to a network 
with an arbitrary degree distribution, one can predict the fraction of nodes required to be randomly removed from the network in order to destroy its LCC (i.e., the critical threshold, $f_{c}$ ) as follows [49];

$$
f_{c}=1-\frac{1}{\left\langle k^{2}\right\rangle /\langle k\rangle-1}
$$

In contrast, the critical threshold of an Erdős-Rényi network (i.e., a randomly wired network) is given by [17]

$$
f_{c}^{\mathrm{ER}}=1-\frac{1}{\langle k\rangle} .
$$

A network is considered to display enhanced robustness if its critical threshold is higher than that of a randomly wired network of the same size (in terms of the number of nodes and links). The UCR-SCN is characterised by $\langle k\rangle=9.56$ and $\left\langle k^{2}\right\rangle=392.16$. Using the above formulae, we can determine $f_{c}$ to be 0.975 and $f_{c}^{\mathrm{ER}}$ to be 0.895. This implies that, in order to destroy the LCC of the UCR-SCN by fragmenting it into many disconnected components, one would need to remove $97.5 \%$ of the nodes (note that this theoretical prediction is generally in agreement with $f_{c}$ of $95.04 \%$ established through the average of 100 simulations). Also, since $f_{c}>f_{c}^{\mathrm{ER}}$, we can conclude that the UCR-SCN displays enhanced robustness against random failure of firms. Indeed, the enhanced robustness of this SCN against random firm removals manifests owing to its hub structure. Random node removals, by definition, affect nodes irrespective of their degrees. Since scalefree networks, such as the UCR-SCN considered here, comprise predominantly of less connected nodes and a few hubs, the chance of randomly removing a hub is almost negligible. Therefore, random node removals are likely to affect mainly the less connected nodes, which, although numerous, play a limited role in maintaining a network's integrity [17].

5.4.2. Directed SCN. For the DMF-SCNs, we demonstrate a simple and intuitive method which considers the impact of firm failure on the output capability of the SCN. This method can be used by practitioners to establish the robustness of their SCNs and also to compare the robustness of various SCN systems against each other.

Since the above robustness concept for the DMFSCNs depends on the demand profiles at the retailers, it may not provide generalizable insights. Therefore, we have not attempted to investigate the robustness character of the full dataset. However, it is evident from the robustness profile presented for the reference SCN 20 (powerdriven hand tools) that the targeted removal of nodes based on their degree has a drastic impact on the overall network robustness-only $5 \%$ of the firms need to be removed for the entire SCN to be incapable to meet any demand at the retailers. In comparison, the SCN is generally much more robust against random removal of firms-about $70 \%$ of the firms need to be removed randomly, before the SCN is incapable of satisfying any demand at the retailers. The above result highlights the importance of hub nodes, through which the majority of the paths traverse. Therefore, the removal of these nodes will have significant impacts on the ability of SCN to meet the demands.

Sheffi and Rice [50] note the importance of building flexibility and redundancies into SCNs as a way of improving the robustness of these systems. In this regard, parallel supply paths with minimal dependencies could be incorporated into SCNs, so that a disruption in one firm does not impact the operations of the other.

\section{Conclusions and Future Directions}

In general, in both DMF and UCR SCNs, we observed degree distributions which conform to power law indicating the existence of hub/leader firms. In both types of SCNs, disassortative mixing was observed in terms of the degree of firms. Interestingly, majority of DMF SCNs also showed disassortative mixing in terms of firm cost and time attributes. Since DMF SCNs are characterised by various functional tiers, no clustering was evident in these systems, while some level of clustering was identified in the UCR SCN. The node centrality correlation assessment carried out against firm attributes (cost and time) for DMF-SCNs reveals a relationship between the position and the function of firms within the system.

Additionally, we identified that the UCR SCN included enhanced topological robustness against random firm failures and self-organized topological features in terms of both betweenness and closeness of firms. However, selforganized features were only present in some of the DMF SCNs.

Finally, we have developed a simple and intuitive method for assessing the robustness of DMF SCNs considering the demand loss at retailers as firms are removed. This analysis outlines the importance of hub firms, through which the majority of the supply paths traverse. Therefore, the removal of these nodes will have significant impacts on the ability of SCN to meet the demands. The robustness score concept can indeed be used to identify the most critical firms in a DMF SCN, which will provide insights beyond the purely topology-based centrality metrics. Future research could investigate the application of TC-DPR we introduced here to identify the configuration, of a particular tiered SCN, which will maximise the robustness score by allocating supply paths based on demand levels at retailers.

Our work for the first time attempted to generalize the topological features of a large number of SCNs from the manufacturing sector. It is notable that since we only considered relatively large networks, finite size effects are minimal. While some topological features were indeed network-specific, the topological similarities between the networks were striking. Therefore, this work could be used as a benchmark for developing generalized growth mechanisms for SCNs in future. 


\section{Appendix}

\section{A. Network and Node-Level Metrics}

TABLE 5: Network-level metrics used and their SCN implications.

\begin{tabular}{lc}
\hline Mathematical representation & SCN implication \\
\hline $\begin{array}{l}\text { For an undirected network: } \\
<k>=1 / N \sum_{i=1}^{N} k_{i}=2 L / N\end{array}$ & Average degree \\
$\begin{array}{l}\text { For a directed network: } \\
<k^{\text {in }}>=1 / N \sum_{i=1}^{N} k_{i}^{\text {in }}=<k^{\text {out }}>=1 / N \sum_{i=1}^{N} k_{i}^{\text {out }}=L / N,\end{array}$ & $\begin{array}{c}\text { Indicates, on average, how many connections a given firm has. } \\
\text { A higher average degree implies good interconnectivity } \\
\text { where } L \text { and } N \text { are the total number of links and }\end{array}$ \\
nodes in the network. & among the firms in the SCN.
\end{tabular}

Network diameter

diameter $=\max _{i, j} l(i, j)$,

where $l$ is the number of hops traversed along the shortest path from node $i$ to $j$.
The diameter of a SCN is the largest distance between any two firms in the network (i.e., the maximum shortest path length). More complex manufacturing processes can include large network diameters (i.e., many stages of production) indicating difficulty in governing the overall SCN under a centralised authority.

Network density $(D)$

Density of a SCN indicated the level of interconnectivity between the firms involved. SCNs with high density indicate good levels of connectivity between firms which can be favourable in terms of efficient information exchange and improved robustness due to redundancy and flexibility [50]. where $\langle k\rangle$ is the mean degree of all the nodes and $N$ is the total number of nodes, in the network.

\section{Network centralisation $(C)$}

Network centralisation provides a value for a given SCN between 0 (if all firms in the SCN have the same connectivity) and 1 (if

the SCN has a star topology). This indicates how the operational

$C=(N / N-2)(\max (k) / N-1-$ density $)$, where $N$ is the total number of nodes in the network and max $(k)$ is the maximum degree of a node within the network. Density is determined as per the equation below. authority is concentrated in a few central firms within the SCN. Highly centralised SCNs can have convenience in terms of centralised decision implementation and high level of

controllability in production planning. However, highly centralised SCNs lack local responsiveness since relationships between firms in various tiers are decoupled [23].

\section{Network heterogeneity $(H)$}

$H=\sqrt{\operatorname{variance}(k)} /<k>$,

where $\langle k>$ is the mean degree and variance $(k)$

is the variance of the degree, of all the nodes in the network.
Heterogeneity is the coefficient of variation of the connectivity. Highly heterogeneous SCNs exhibit hub firms (i.e., firms with high number of contractual connections). In extreme cases, there may be many super

large hubs (winner-take-all scenario, indicating centralised control of the overall SCN through a very few firms).

\section{Average clustering coefficient $(<C>)$}

$<C>=\sum_{i} C_{i} / N$,

where $N$ is the total number of nodes in the network and $C_{i}$ is the number of triangles connected to node $i$ divided by the number of triples centered around node $i$.
The clustering coefficient indicates the degree to which firms in a SCN tend to cluster together around a given firm. For example, it can indicate how various suppliers behave with respect to the final assembler at the global level [23]. Therefore, the higher the clustering coefficient, the more dependent suppliers are on each other for production [46].

Characteristic (or average) path length $(<l>)$

The characteristic path length $<l>$ is $<l>=1 / N(N-1) \sum_{i \neq j} l_{i, j}$,

where $N$ is the total number of nodes in the network and $l_{i, j}$ is the shortest topological distance between nodes $i$ and $j$.

The degree distribution $P_{k}$ of an undirected scale-free network is approximated with power law as follows: $P_{k} \sim k^{-\gamma}$, where $k$ is the degree of the node and $\gamma$ is the degree
Characteristic (or average) path length is the average topological distance between all pairs of firms (along the shortest path) in a SCN. It measures how efficiently information can be transferred between pairs of firms within a SCN.
SCNs with $\gamma<2$ include very large hubs which acquire control through contractual relationships with other firms at a rate faster than the growth of the SCN in terms of new firm additions. As $\gamma$ continues to increase beyond 2, the SCNs include smaller and less numerous hubs, which 
TABLE 5: Continued.

\begin{tabular}{l}
\hline Mathematical representation \\
$\begin{array}{l}\text { exponent (also known as the power law or scale-free exponent). Directed } \\
\text { networks generally include two separate degree distributions, one for the } \\
\text { in-degree and another for the out-degree. }\end{array}$ \\
$\begin{array}{l}\text { In such cases, there will be two separate degree exponents, } \\
\text { i.e., } \gamma_{\text {in }} \text { and } \gamma_{\text {out }} \text { all firms have almost the same number of connections. In particular, } \\
\text { when } \gamma \text { is less than or equal to 2, the network topology is referred to as a } \\
\text { "hub and spoke" topology; }\end{array}$ \\
$\begin{array}{c}\text { when } \gamma \text { is higher than } 2 \text { but less than 3, the network topology is } \\
\text { referred to as scale-free; and when } \gamma \text { is higher than 3, the } \\
\text { network topology is random. }\end{array}$ \\
\hline
\end{tabular}

Assortativity $(\rho)[36]$

Assortativity is formally defined as a correlation function of excess degree distributions and link distribution of a network. For undirected networks, when degree distribution is denoted as $p_{k}$ and excess degree (remaining degree) distribution is denoted as $q_{k}$, one can introduce the quantity $e_{j, k}$ as the joint probability distribution of the remaining degree distribution of the remaining degrees of the two nodes at either end of a randomly chosen link.

Given these distributions, the assortativity of an undirected network is defined as

$\rho=1 / \sigma_{q}^{2}\left[\sum_{j k} j k\left(e_{j k}-q_{j} q_{k}\right)\right]$,

where $\sigma_{q}$ is the standard deviation of $q_{k}$ which is given as

$\sigma_{q}^{2}=\sum_{k} k^{2} q_{k}-\left[\sum_{k} k q_{k}\right]^{2}$.

Assortativity, $\rho$ is a value between -1 and 1 . For $\rho>0$, the network is assortative; for $\rho=0$, the network is neutral; and for $\rho<0$, the network is disassortative.
Positive assortativity means that the firms with similar connectivity would have a higher tendency to connect with each other (for example, highly connected firms could be managing subcommunities in certain areas of production and then connect to other high-degree firms undertaking the same function). This structure can lead to cascading disruptions-where a disruption at one leaf node can spread quickly within the network through the connected hubs [46]. In contrast, a negative assortativity indicates that it is the firms with dissimilar connectivity that tend to pair up in the given network.

Note that assortativity can also be defined in terms of node attributes other than the degree.

TABLE 6: Node-level metrics used and their SCN implications.

Mathematical representation

SCN implication

Degree $(k)$

Represents the number of direct neighbours (connections) a given firm has. For instance, in a given SCN, the firm with the highest degree (such as the integrators that assemble components) is deemed to have the

In undirected networks, the degree of node $i$ is given as largest impact on operational decisions and strategic behaviours of other $k_{i}=\sum_{j=1}^{N} A_{j i}=\sum_{i=1}^{N} A_{j i}$. firms in that particular SCN. Such a firm has the power to reconcile the In directed networks, the degree of node $i$ is separated into in- and outdegrees, as follows:

$k_{i}^{\text {in }}=\sum_{j=1}^{N} A_{i j}, \quad k_{i}^{\text {out }}=\sum_{j=1}^{N} A_{j i}$,

where $A_{i j}$ is any element of the adjacency matrix $A$.

differences between various other firms in the SCN and align their efforts with greater SCN goals [23].

In directed networks, the firms which have high in-degree are considered to be "integrators" who collect information from various other firms to create high-value products. In contrast, the firms which have high outdegree are considered to be "allocators" who are generally responsible for distribution of high-demand resources to other firms and/or customers.

Betweenness centrality (normalised) [51]

The betweenness centrality of a node $n$ is defined as

$C_{b}(n)=2 /(N-1)(N-2) \sum_{s \neq n \neq t}\left(\sigma_{s, t}(n) / \sigma_{s, t}\right)$,

where $s$ and $t$ are nodes in the network, which are different from $n, \sigma_{s, t}$ denotes the number of shortest paths from $s$ to $t$, and $\sigma_{s, t}(n)$ is the number of shortest paths from $s$ to $t$ that $n$ lies on.
Betweenness centrality of a firm is the number of shortest path relationships going through it, considering the shortest path relationships that connect any two given firms in the SCN. Therefore, it indicates the extent to which a firm can intervene over interactions among other firms in the SCN by being a gatekeeper for relationships [23]. Those firms with high levels of betweenness generally play a vital role in SCNs-mainly owing to their ability to increase the overall efficiency of the SCN by smoothing various exchange processes between firms.

Closeness centrality [52]

The closeness centrality of a node $n$ is defined as $C_{c}(n)=1 /<L(n, m)>$, where $\langle L(n, m)>$ is the length of the shortest path between two nodes $n$ and $m$ (note that for unweighted graphs with no geodesic distance information, each link is assumed to be one unit of distance). The closeness centrality of each node is a number between 0 and 1 .
Closeness centrality is a measure of the time that it takes to spread the information from a particular firm to the other firms in the network. While it is closely related to betweenness centrality, closeness is more relevant in situations where a firm acts as a generator of information rather than a mere mediator/gatekeeper. Firms with high closeness centrality levels enable the overall SCN to be more market sensitive (i.e., responsive) by spreading the actual market demand information with the other upstream firms [9]. 


\section{B. Node-Level Centrality Correlations with}

Stage Time and Stage Cost

B.1 Correlation Analysis for Total Degree.

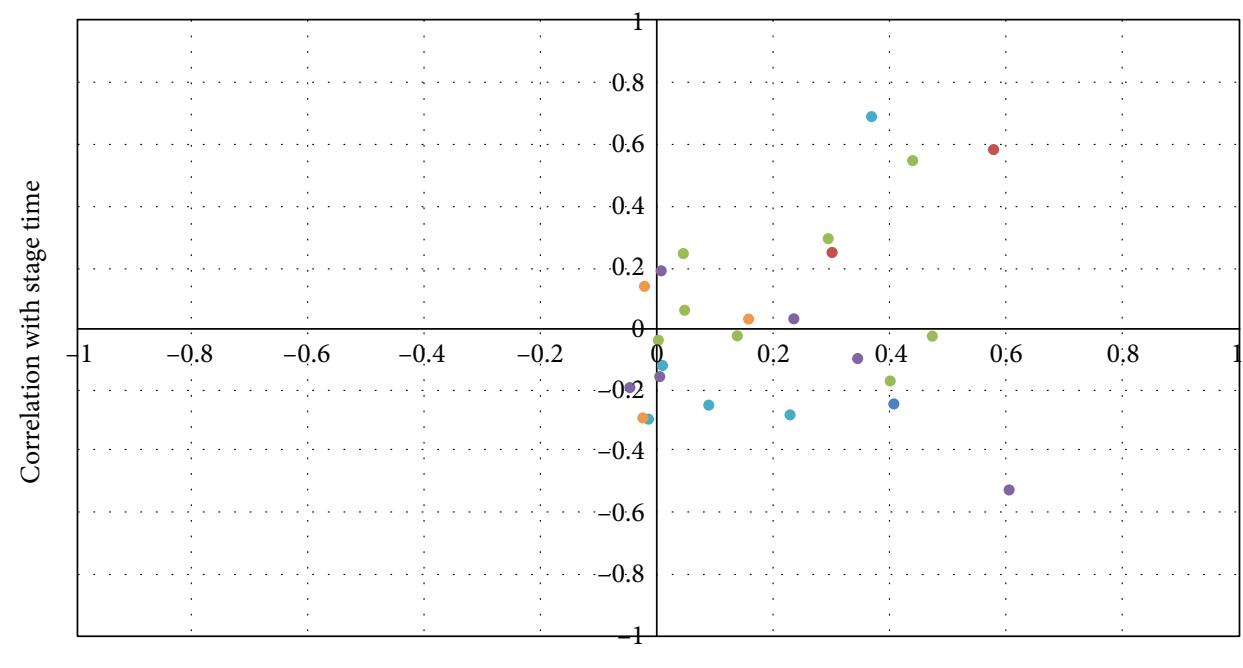

Correlation with stage cost

- Aircraft engines and engine parts

- Arrangement of transportation of freight and cargo

- Chemical
- Computer-related

- Electrical

- Farm machinery and equipment

FIgURE 10

B.2 Correlation Analysis for In-Degree.

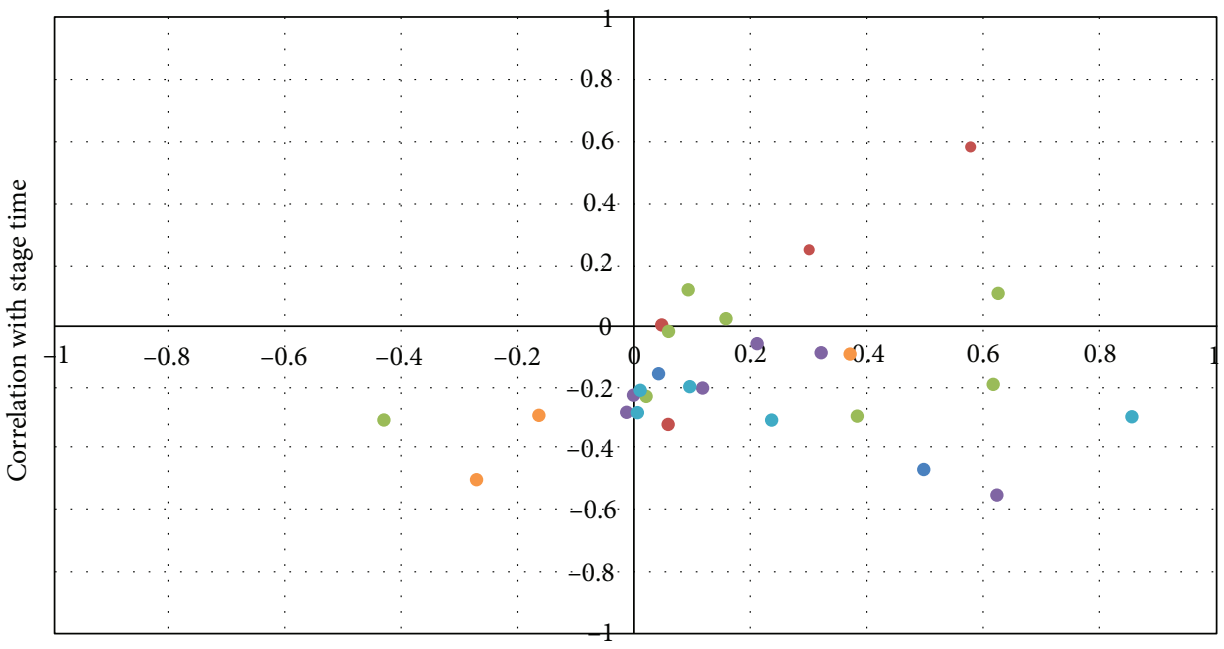

Correlation with stage cost

- Aircraft engines and engine parts

- Arrangement of transportation of freight and cargo

- Chemical
- Computer-related

- Electrical

- Farm machinery and equipment

FIgURe 11 
B.3 Correlation Analysis for Out-Degree.

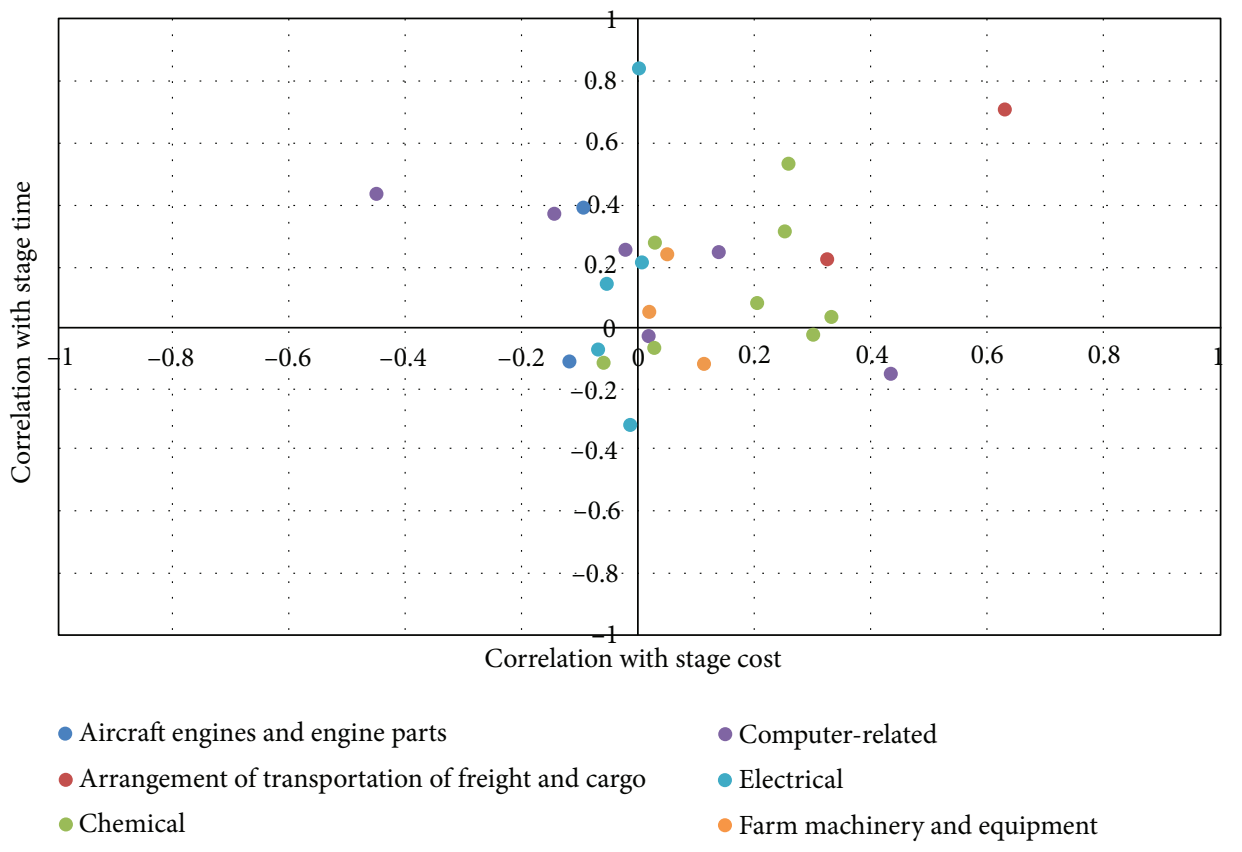

FIGURE 12

B.4 Correlation Analysis for Betweenness Centrality.

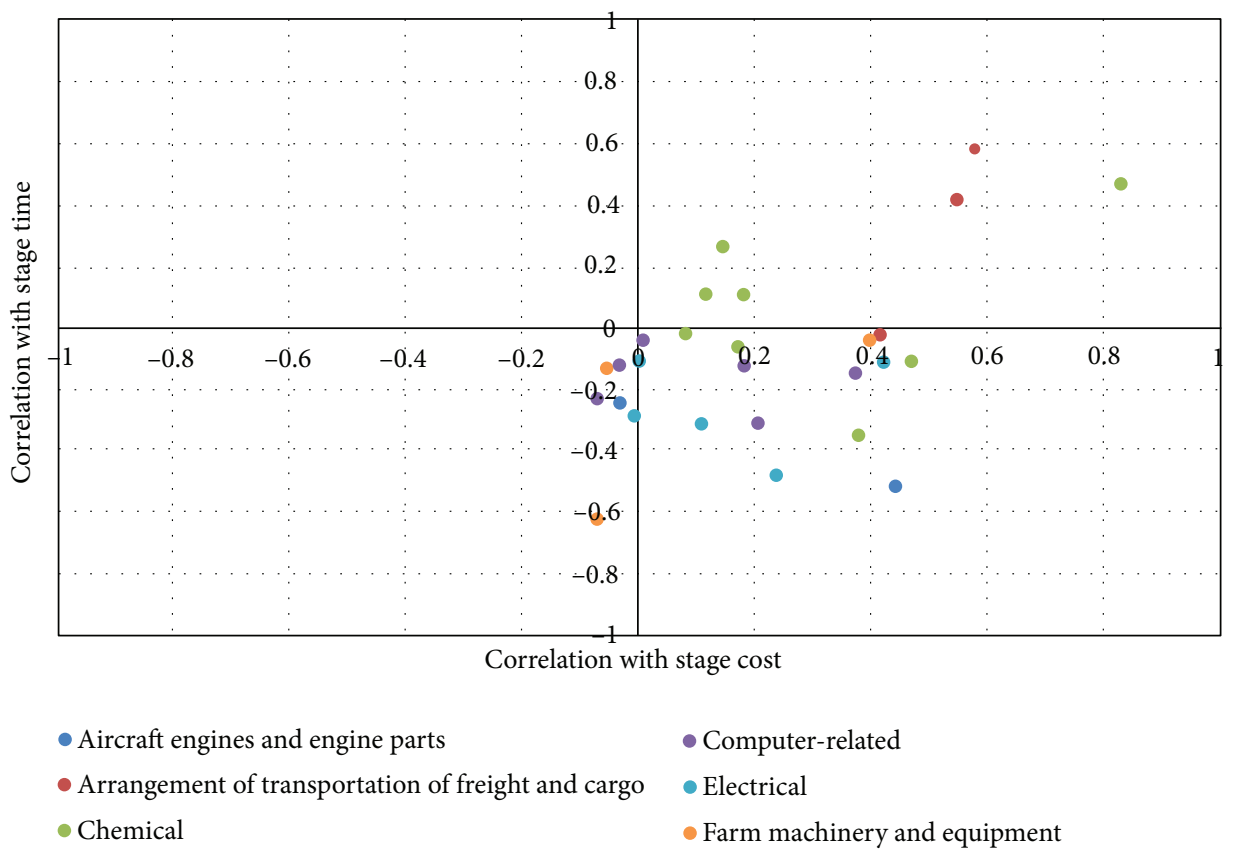

FIgURE 13 
B.5 Correlation Analysis for Closeness Centrality.

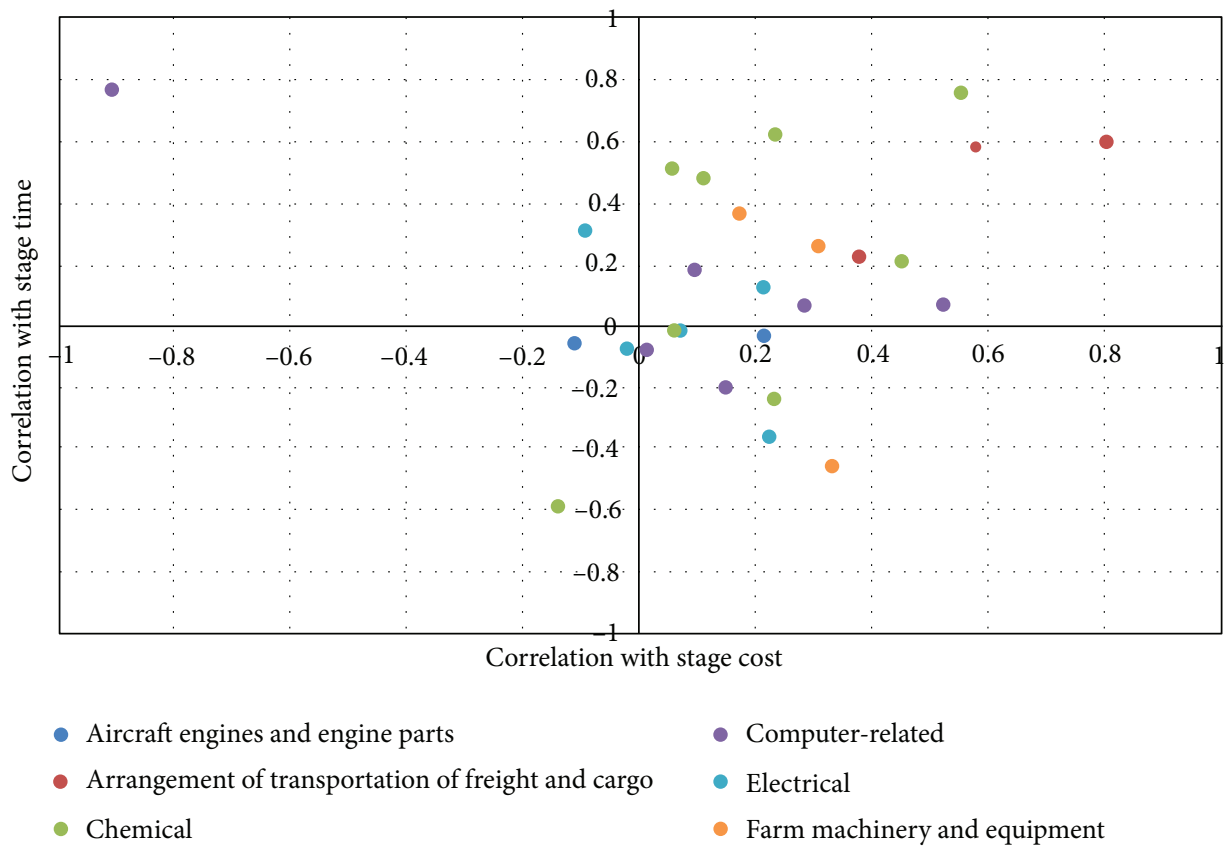

FIGURE 14

\section{Data Availability}

The DMF SCN dataset is publicly available under the published work of Willems [14]. The adjacency matrix for the UCR SCN can be provided upon request by the corresponding author.

\section{Conflicts of Interest}

The authors declare that there is no conflict of interest regarding the publication of this paper.

\section{Acknowledgments}

The authors acknowledge the Australian Research Council (ARC) for funding this work under grant DP140103643.

\section{References}

[1] T. Y. Choi, K. J. Dooley, and M. Rungtusanatham, "Supply networks and complex adaptive systems: control versus emergence," Journal of Operations Management, vol. 19, no. 3, pp. 351-366, 2001.

[2] S. D. Pathak, J. M. Day, A. Nair, W. J. Sawaya, and M. M. Kristal, "Complexity and adaptivity in supply networks: building supply network theory using a complex adaptive systems perspective*," Decision Sciences, vol. 38, no. 4, pp. 547-580, 2007.

[3] H. P. Thadakamalla, U. N. Raghavan, S. Kumara, and A. Albert, "Survivability of multiagent-based supply networks: a topological perspective," IEEE Intelligent Systems, vol. 19, no. 5, pp. 24-31, 2004.

[4] Y. Kim, Y. S. Chen, and K. Linderman, "Supply network disruption and resilience: a network structural perspective," Journal of Operations Management, vol. 33-34, pp. 43-59, 2015.

[5] Y. Li, "Networked analysis approach of supply chain network," Journal of Networks, vol. 9, no. 3, 2014.

[6] S. I. Mari, Y. H. Lee, M. S. Memon, Y. S. Park, and M. Kim, "Adaptivity of complex network topologies for designing resilient supply chain networks," International Journal of Industrial Engineering, vol. 22, no. 1, pp. 102-116, 2015.

[7] L. Wen, M. F. Guo, and L. J. wang, "Statistic characteristics analysis of directed supply chain complex network," International Journal of Advancements in Computing Technology, vol. 4, no. 21, pp. 84-91, 2012.

[8] M. Xu, X. Wang, and L. Zhao, "Predicted supply chain resilience based on structural evolution against random supply disruptions," International Journal of Systems Science: Operations \& Logistics, vol. 1, no. 2, pp. 105-117, 2014.

[9] N. R. Xu, J. B. Liu, D. X. Li, and J. Wang, "Research on evolutionary mechanism of agile supply chain network via complex network theory," Mathematical Problems in Engineering, vol. 2016, Article ID 4346580, 9 pages, 2016.

[10] Q. Xuan, F. Du, Y. Li, and T. J. Wu, "A framework to model the topological structure of supply networks," IEEE Transactions on Automation Science and Engineering, vol. 8, no. 2, pp. 442-446, 2011.

[11] K. Zhao, A. Kumar, and J. Yen, "Achieving high robustness in supply distribution networks by rewiring," IEEE Transactions on Engineering Management, vol. 58, no. 2, pp. 347-362, 2011. 
[12] K. Zhao, A. Kumar, T. P. Harrison, and J. Yen, "Analyzing the resilience of complex supply network topologies against random and targeted disruptions," IEEE Systems Journal, vol. 5, no. 1, pp. 28-39, 2011.

[13] M. Parhi, "Impact of the changing facets of inter-firm interactions on manufacturing excellence: a social network perspective of the Indian automotive industry," Asian Journal of Technology Innovation, vol. 16, no. 1, pp. 117-141, 2008.

[14] S. P. Willems, "Data set-real-world multiechelon supply chains used for inventory optimization," Manufacturing \& Service Operations Management, vol. 10, no. 1, pp. 19-23, 2008.

[15] S. Lazzarini, F. Chaddad, and M. Cook, "Integrating supply chain and network analyses: the study of netchains," Journal on Chain and Network Science, vol. 1, no. 1, pp. 7-22, 2001.

[16] E. J. S. Hearnshaw and M. M. J. Wilson, "A complex network approach to supply chain network theory," International Journal of Operations \& Production Management, vol. 33, no. 4, pp. 442-469, 2013.

[17] A. Barabási, Network Science, Cambridge University Press, 2016.

[18] S. M. Park and B. J. Kim, "Dynamic behaviors in directed networks," Physical Review E, vol. 74, no. 2, article 026114, 2006.

[19] N. Schwartz, R. Cohen, D. Ben-Avraham, A. L. Barabási, and S. Havlin, "Percolation in directed scale-free networks," Physical Review E, vol. 66, no. 1, article 015104, 2002.

[20] L. D. F. Costa, F. A. Rodrigues, G. Travieso, and P. R. Villas Boas, "Characterization of complex networks: a survey of measurements," Advances in Physics, vol. 56, no. 1, pp. 167-242, 2007.

[21] M. Rubinov and O. Sporns, "Complex network measures of brain connectivity: uses and interpretations," NeuroImage, vol. 52, no. 3, pp. 1059-1069, 2010.

[22] S. Perera, M. G. H. Bell, and M. C. J. Bliemer, "Network science approach to modelling the topology and robustness of supply chain networks: a review and perspective," Applied Network Science, vol. 2, no. 1, p. 33, 2017.

[23] Y. Kim, T. Y. Choi, T. Yan, and K. Dooley, "Structural investigation of supply networks: a social network analysis approach," Journal of Operations Management, vol. 29, no. 3, pp. 194-211, 2011.

[24] T. Y. Choi and Y. Hong, "Unveiling the structure of supply networks: case studies in Honda, Acura and DaimlerChrysler," Journal of Operations Management, vol. 20, no. 5, pp. 469-493, 2002.

[25] T. Kito, A. Brintrup, S. New, and F. Reed-Tsochas, The Structure of the Toyota Supply Network: An Empirical Analysis, SSRN Scholarly Paper ID 2412512, Social Science Research Network, Rochester, NY, 2014.

[26] A. Brintrup, Y. Wang, and A. Tiwari, "Supply networks as complex systems: a network-science-based characterization," IEEE Systems Journal, vol. 11, no. 4, pp. 2170-2181, 2017.

[27] P. Orenstein, "How does supply network evolution and its topological structure impact supply chain performance?," in 2016 Second International Symposium on Stochastic Models in Reliability Engineering, Life Science and Operations Management (SMRLO), pp. 562-569, Beersheba, Israel, February 2016.

[28] S. Maslov and K. Sneppen, "Specificity and stability in topology of protein networks," Science, vol. 296, no. 5569, pp. 910-913, 2002.
[29] R. Noldus and P. Van Mieghem, "Assortativity in complex networks," Journal of Complex Networks, vol. 3, no. 4, pp. 507-542, 2015.

[30] B. K. Fosdick, D. B. Larremore, J. Nishimura, and J. Ugander, "Configuring random graph models with fixed degree sequences," 2016, http://arxiv.org/abs/1608.00607.

[31] C. Diebolt, T. Mishra, and M. Parhi, "Dynamics of distribution and diffusion of new technology," in India Studies in Business and Economics ReDIF-Book, Springer, 2016.

[32] T. Becker, M. Meyer, and K. Windt, "A manufacturing systems network model for the evaluation of complex manufacturing systems," International Journal of Productivity and Performance Management, vol. 63, no. 3, pp. 324-340, 2014.

[33] Z. Gang, Y. Ying-Bao, B. Xu, and P. Qi-Yuan, "On the topological properties of urban complex supply chain network of agricultural products in mainland China," Transportation Letters, vol. 7, no. 4, pp. 188-195, 2015.

[34] W. Keqiang, Z. Zhaofeng, and S. Dongchuan, "Structure analysis of supply chain networks based on complex network theory," in 2008 Fourth International Conference on Semantics, Knowledge and Grid, pp. 493-494, Beijing, China, December 2008.

[35] A. L. Barabási and R. Albert, "Emergence of scaling in random networks," Science, vol. 286, no. 5439, pp. 509-512, 1999.

[36] M. E. J. Newman, “Assortative mixing in networks," Physical Review Letters, vol. 89, no. 20, article 208701, 2002.

[37] M. E. J. Newman, "The structure and function of complex networks,” SIAM Review, vol. 45, no. 2, pp. 167-256, 2003.

[38] C. Bedogne' and G. J. Rodgers, "Complex growing networks with intrinsic vertex fitness," Physical Review E, vol. 74, no. 4, article 046115, 2006.

[39] G. Bianconi and A. L. Barabási, "Competition and multiscaling in evolving networks," Europhysics Letters (EPL), vol. 54, no. 4, pp. 436-442, 2001.

[40] G. Caldarelli, A. Capocci, P. De Los Rios, and M. A. Munoz, "Scale-free networks from varying vertex intrinsic fitness," Physical Review Letters, vol. 89, no. 25, article 258702, 2002.

[41] S. Perera, M. G. H. Bell, and M. C. J. Bliemer, "Resilience characteristics of supply network topologies generated by fitness based growth models," Transportation Research Board 95th Annual Meeting, 2016, Washington DC, United States, January $2016,2016$.

[42] K. Nguyen and D. A. Tran, "Fitness-based generative models for power-law networks," in Handbook of Optimization in Complex Networks, pp. 39-53, Springer, Boston, MA, 2012.

[43] M. Bell, S. Perera, M. Piraveenan, M. Bliemer, T. Latty, and C. Reid, "Network growth models: a behavioural basis for attachment proportional to fitness," Scientific Reports, vol. 7, no. $1,2017$.

[44] S. J. Hu, X. Zhu, H. Wang, and Y. Koren, "Product variety and manufacturing complexity in assembly systems and supply chains," CIRP Annals, vol. 57, no. 1, pp. 45-48, 2008.

[45] C. Song, S. Havlin, and H. A. Makse, "Origins of fractality in the growth of complex networks," Nature Physics, vol. 2, no. 4, pp. 275-281, 2006.

[46] A. Brintrup, A. Ledwoch, and J. Barros, "Topological robustness of the global automotive industry," Logistics Research, vol. 9, no. 1, 2016.

[47] T. Y. Choi and Z. Wu, "Taking the leap from dyads to triads: buyer-supplier relationships in supply networks," Journal of Purchasing and Supply Management, vol. 15, no. 4, pp. 263266, 2009. 
[48] S. P. Borgatti and X. Li, "On social network analysis in a supply chain context*," Journal of Supply Chain Management, vol. 45, no. 2, pp. 5-22, 2009.

[49] R. Cohen, K. Erez, D. Ben-Avraham, and S. Havlin, "Resilience of the Internet to random breakdowns," Physical Review Letters, vol. 85, no. 21, pp. 4626-4628, 2000.

[50] Y. Sheffi and J. B. Rice Jr, “A supply chain view of the resilient enterprise," MIT Sloan Management Review, vol. 47, no. 1, p. 41, 2005.

[51] L. C. Freeman, "A set of measures of centrality based on betweenness," Sociometry, vol. 40, no. 1, pp. 35-41, 1977.

[52] G. Sabidussi, "The centrality index of a graph," Psychometrika, vol. 31, no. 4, pp. 581-603, 1966. 


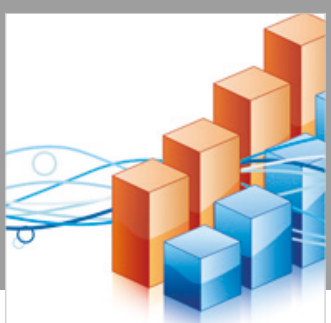

Advances in

Operations Research

\section{-n-m}
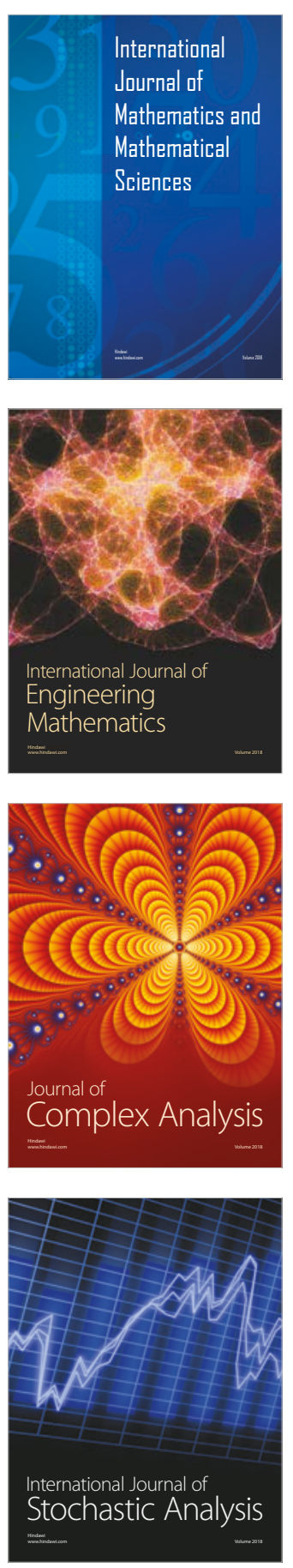
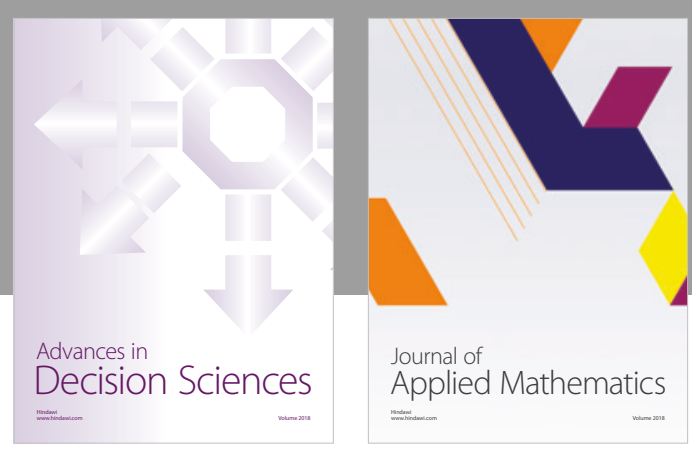

Journal of

Applied Mathematics
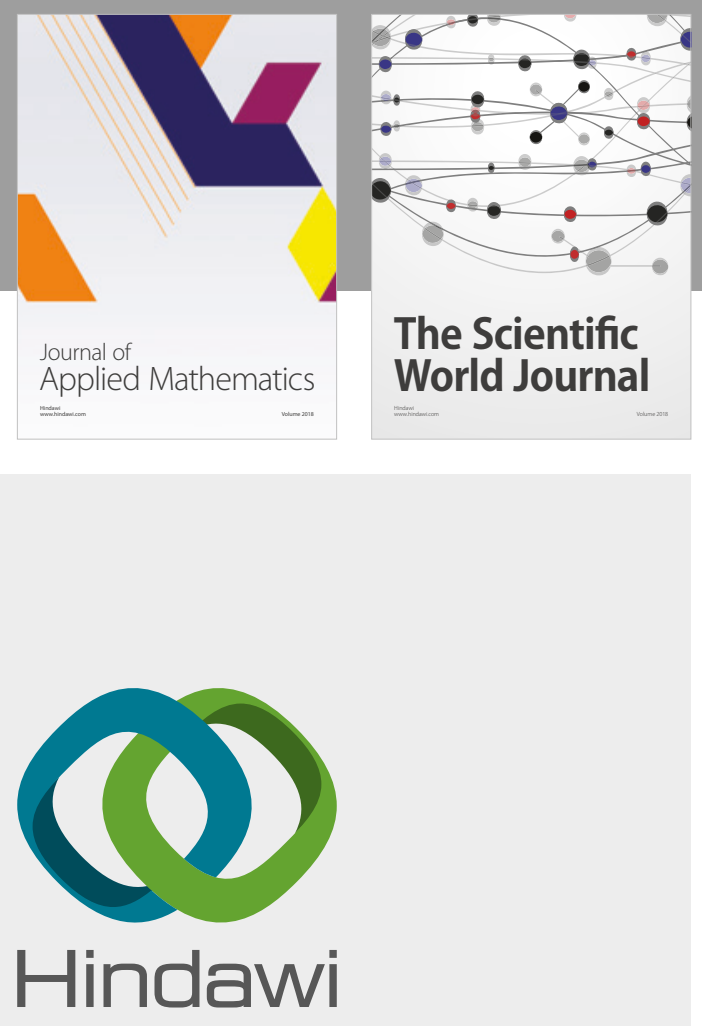

Submit your manuscripts at

www.hindawi.com

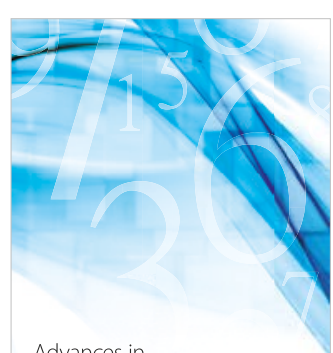

Advances in
Numerical Analysis
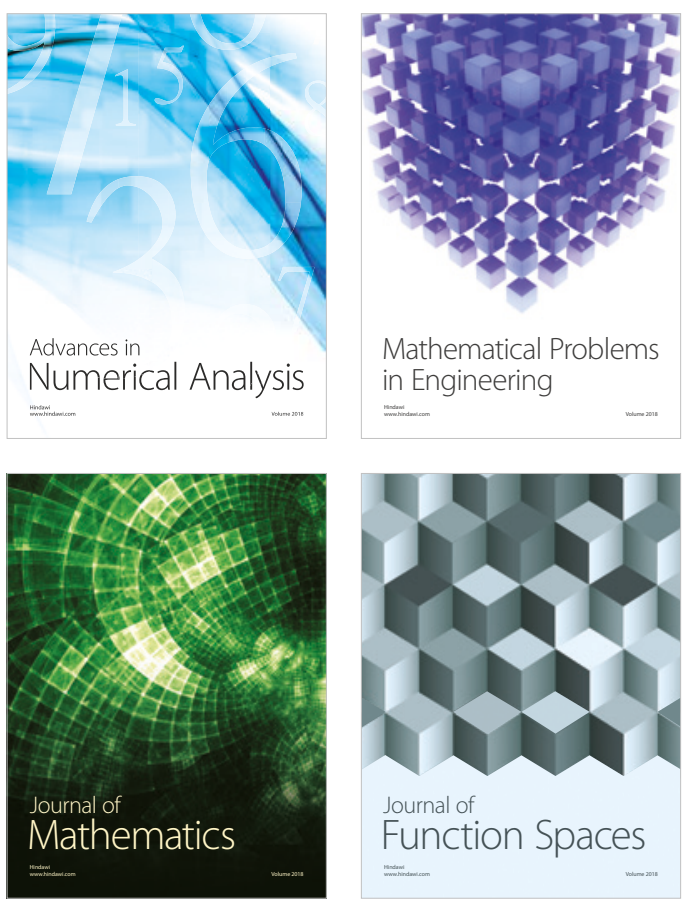

Mathematical Problems in Engineering

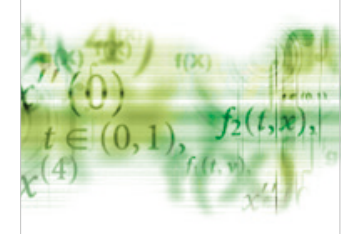

International Journal of

Differential Equations

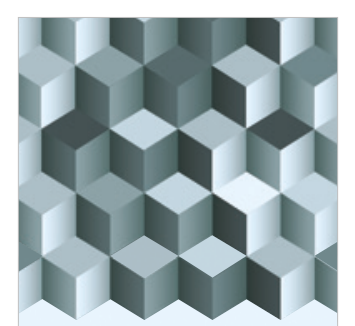

Journal of

Function Spaces
The Scientific

World Journal

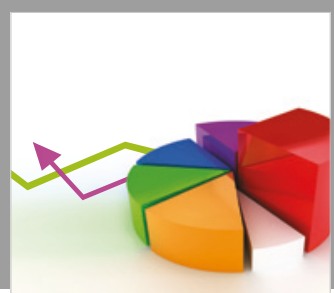

Journal of

Probability and Statistics
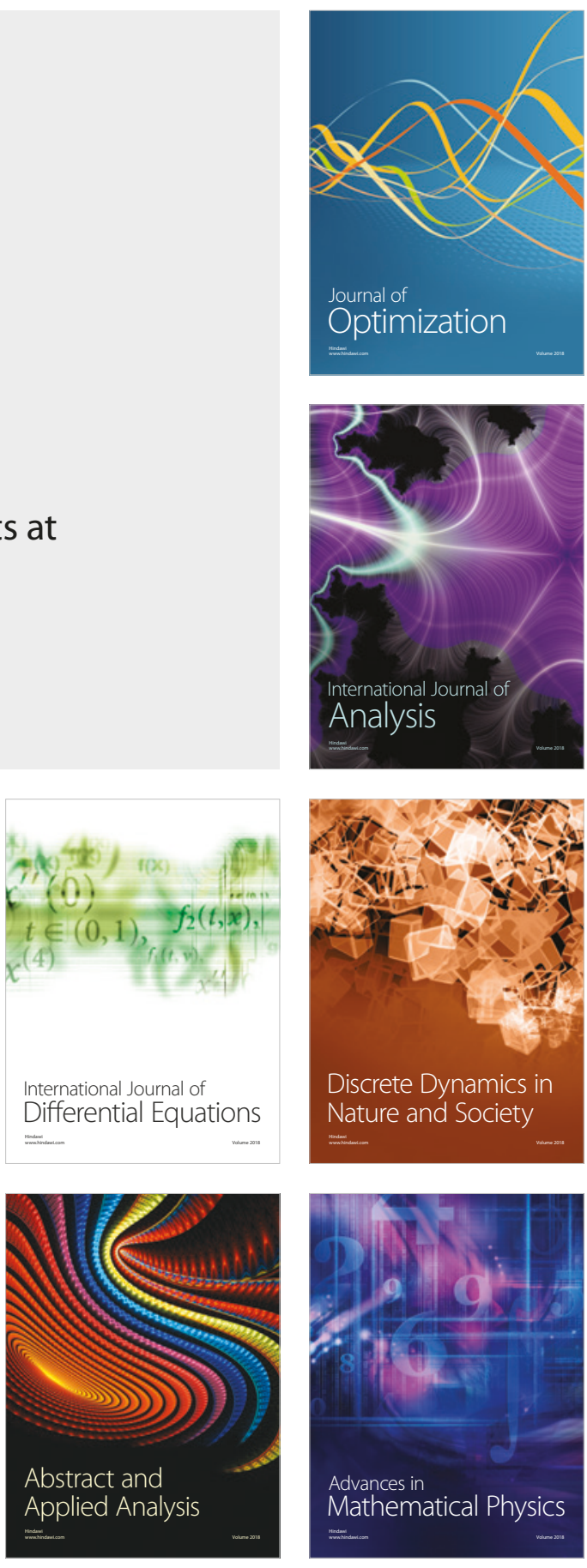